\title{
High Performance Computational Models of Synthetic Jet Actuators for Step Change Improvements in Freight Efficiency
}

Approved for public release. Distribution is unlimited.
David Pointer

Marc-Olivier Delchini

Emilian Popov

David Menicovich

Michael Amitay

August, 2018 


\section{DOCUMENT AVAILABILITY}

Reports produced after January 1, 1996, are generally available free via US Department of Energy (DOE) SciTech Connect.

Website: www.osti.gov/

Reports produced before January 1, 1996, may be purchased by members of the public from the following source:

National Technical Information Service

5285 Port Royal Road

Springfield, VA 22161

Telephone: 703-605-6000 (1-800-553-6847)

TDD: $703-487-4639$

Fax: 703-605-6900

E-mail: info@ntis.gov

Website: http://classic.ntis.gov/

Reports are available to DOE employees, DOE contractors, Energy Technology Data Exchange representatives, and International Nuclear Information System representatives from the following source:

Office of Scientific and Technical Information

PO Box 62

Oak Ridge, TN 37831

Telephone: 865-576-8401

Fax: 865-576-5728

E-mail: report@osti.gov

Website: http://www.osti.gov/contact.html

This report was prepared as an account of work sponsored by an agency of the United States Government. Neither the United States Government nor any agency thereof, nor any of their employees, makes any warranty, express or implied, or assumes any legal liability or responsibility for the accuracy, completeness, or usefulness of any information, apparatus, product, or process disclosed, or represents that its use would not infringe privately owned rights. Reference herein to any specific commercial product, process, or service by trade name, trademark, manufacturer, or otherwise, does not necessarily constitute or imply its endorsement, recommendation, or favoring by the United States Government or any agency thereof. The views and opinions of authors expressed herein do not necessarily state or reflect those of the United States Government or any agency thereof. 
Reactor and Nuclear Systems Division

\title{
High Performance Computational Models of Synthetic Jet Actuators for Step Change Improvements in Freight Efficiency
}

\author{
Author(s) \\ David Pointer \\ Marc-Olivier Delchini \\ Emilian Popov \\ David Menicovich \\ Michael Amitay
}

Date Published: August, 2018

Prepared by

OAK RIDGE NATIONAL LABORATORY

Oak Ridge, TN 37831-6283

managed by

UT-Battelle, LLC

for the

US DEPARTMENT OF ENERGY

under contract DE-AC05-00OR22725 



\section{CONTENTS}

LIST OF FIGURES $\ldots \ldots \ldots \ldots \ldots \ldots \ldots \ldots \ldots \ldots \ldots$

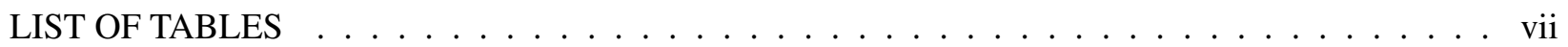

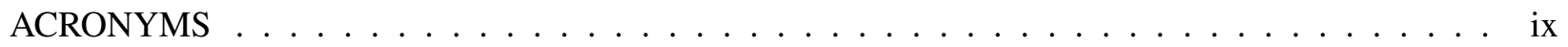

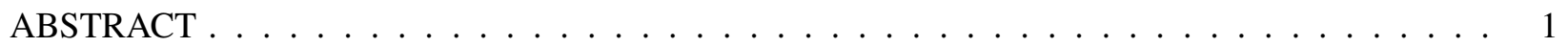

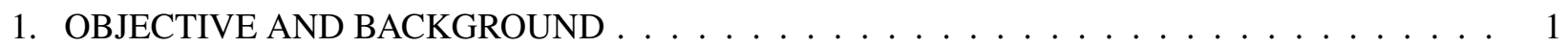

2. BENEFITS TO THE DOE MISSION $\ldots \ldots \ldots \ldots \ldots \ldots \ldots \ldots \ldots$

3. TECHNICAL DISCUSSION OF WORK PERFORMED . . . . . . . . . . . . . . . . . . 2

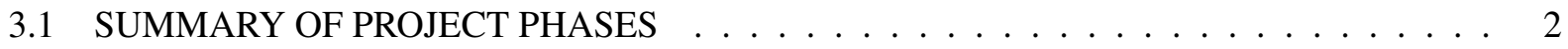

3.2 Initial CFD Model Development . . . . . . . . . . . . . . . . . 2

3.3 Design Exploration Analyses . . . . . . . . . . . . . . . . . . . . . 3

3.4 Practical Implementation, Model calibration to Displacement Data and Preliminary drag

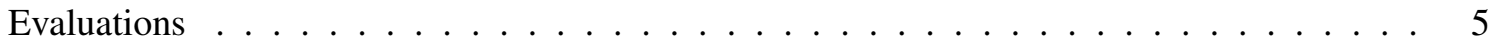

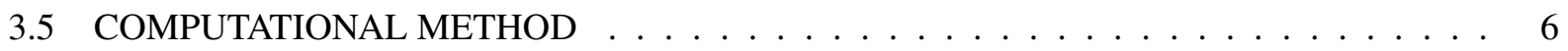

3.6 NON-DIMENSIONALIZATION OF THE INCOMPRESSIBLE NAVIER-STOKES

EQUATIONS FOR A SYNTHETIC JET . . . . . . . . . . . . . . . . . . . 8

3.6.1 Non-dimensionalization of the incompressible Navier-Stokes equations with

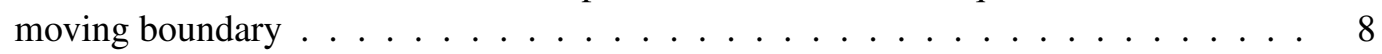

3.6.2 Reference velocity for an actuator . . . . . . . . . . . . . . . 9

3.6.3 Derivation of a reference velocity for a 2-D model of an actuator . . . . . . . . . . 11

3.6.4 Derivation of a reference velocity for a 3-D model of an actuator . . . . . . . . . 13

3.6.5 Non-dimensionalized Navier-Stokes equations with moving boundary conditions . . 14

3.6.6 Relationship between the maximum displacement and the membrane frequency . . . 15

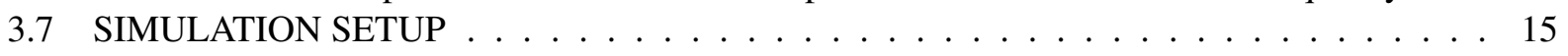

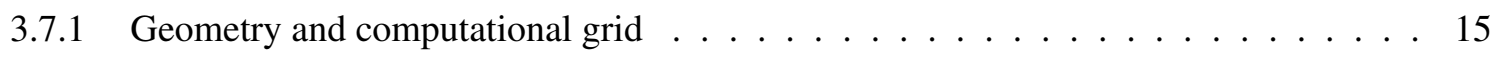

3.7 .2 Initial conditions . . . . . . . . . . . . . . . . . . . . . . 19

3.8 DEFINITION AND AVERAGING . . . . . . . . . . . . . . . . . . . . . . . . . . . 19

3.9 USE OF DIRECT NUMERICAL SIMULATION DATA FOR BENCHMARKING . . . . . 21

3.9 .1 Time-averaged velocity field . . . . . . . . . . . . . . . . 21

3.9.2 Volume-averaged velocity in the nozzle and in the cavity . . . . . . . . . . 23

3.9 .3 Drag in the nozzle . . . . . . . . . . . . . . . . . 25

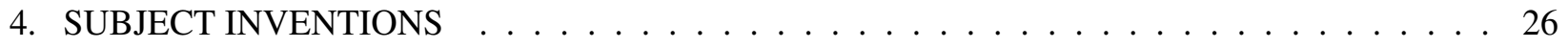

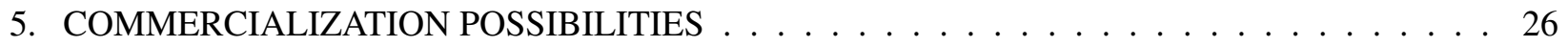

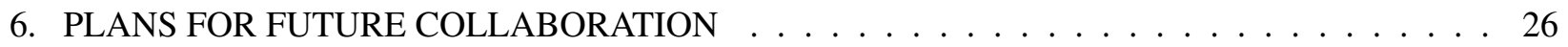

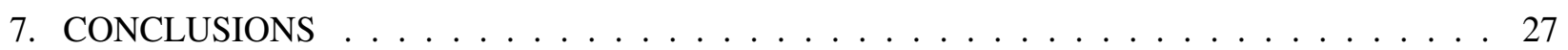





\section{LIST OF FIGURES}

1 Sketch and concept of an actuator $\ldots \ldots \ldots \ldots \ldots$

2 SJA simulation of disk motion and induced jet formation when operated at a frequency of $1,000 \mathrm{~Hz}$ showing the average orifice velocity and vorticity evolution in time for ten cycles of the actuator. The top images are experimental, and the remaining images evaluate different turbulence models (shear stress turbulence (SST), renormalized k-epsilon (RKE), low RKE, and Reynolds-stress turbulence (RST) . . . . . . . . . . . . . . 4

3 Snapshots of detailed velocity profiles across the narrow gap of a SJA operating at 1,000 $\mathrm{Hz}$, as predicted by high-resolution DNS. Time $\mathrm{t}=\mathrm{T}$ aligns with the completion of the compressive stroke ......................... 5

4 Demonstration sensitivity study evaluating impact of simple design changes on the maximum time-averaged velocity of the jet. As should be expected, the jet velocity is strongly correlated to the displacement of the membrane that drives the air flow. . . . . . . 5

5 Disk shapes in the function of the driving frequency for low and high ranges.

Comparison to a sine-shaped profile is provided, and the resultant difference in the displaced volume is calculated. . . . . . . . . . . . . . . . . 7

6 Clipped view of an SJA. Numerical solution obtained with OpenFOAM.3 (left). Slice view of the 3 -D turbulent flow around a car (right) . . . . . . . . . . . . . 7

7 Polynomial fit coefficients for the maximum displacement as a function of the membrane

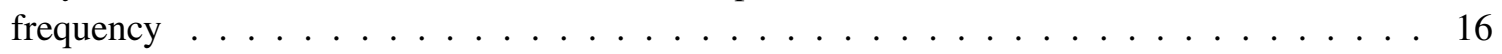

8 Plot of the polynomial fit for the maximum displacement as a function of the membrane

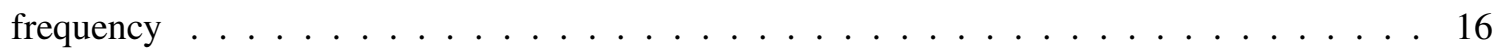

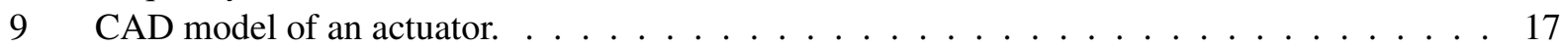

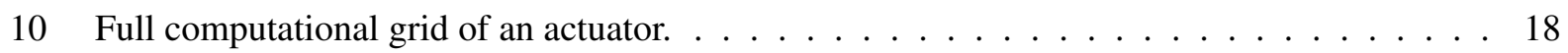

11 Zoom-in of the computational mesh in the nozzle region. . . . . . . . . . . . . . . . 19

12 Time-averaged velocity magnitude over 6 cycles for case 1 in the XY plane. . . . . . . . 22

13 Time-averaged velocity magnitude over 6 cycles for case 1 in the YZ plane. . . . . . . . 23

14 Time-averaged velocity magnitude over 6 cycles for case 1 in the YZ plane. . . . . . . . . 24

16 Surface-averaged drag in the nozzle. . . . . . . . . . . . . . 25 



\section{LIST OF TABLES}

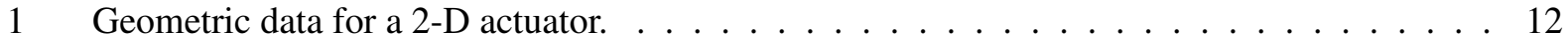

2 Non-dimensional numbers for a 2-D actuator. . . . . . . . . . . . . . . . . 12

3 Geometric data for a 2-D actuator. . . . . . . . . . . . . . . . . . . . 14

4 Nondimensional numbers for a 3-D actuator. . . . . . . . . . . . . . . . . . . . . 14

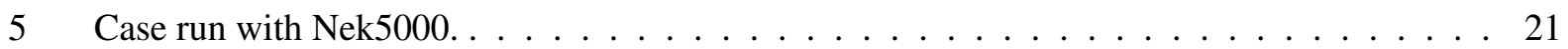

6 Time and surface-averaged drag on nozzle wall for case $1 \ldots \ldots \ldots$ 

ACRONYMS

$\begin{array}{ll}\text { AFC } & \text { Active Flow Control } \\ \text { ALE } & \text { Arbitrary Lagrangian/Eulerian } \\ \text { CAD } & \text { Computer Aided Design } \\ \text { CFD } & \text { Computational Fluid Dynamics } \\ \text { CRADA } & \text { Cooperative research and development agreement } \\ \text { DNS } & \text { Direct Numerical Solution } \\ \text { DOE } & \text { Department Of Energy } \\ \text { GLL } & \text { Gauss-Lobbato-Legendre } \\ \text { HDV } & \text { Heavy Duty Vehicle } \\ \text { INS } & \text { Incompressible Navier Stokes } \\ \text { LES } & \text { Large Eddy Simulation } \\ \text { HPC } & \text { High Performance Computing } \\ \text { ORNL } & \text { Oak Ridge National Laboratory } \\ \text { PoC } & \text { proof of concept } \\ \text { RKE } & \text { Renormalized k-epsilon } \\ \text { RST } & \text { Reynolds-stress turbulence } \\ \text { SST } & \text { Shear Stress Turbulence } \\ \text { SEM } & \text { Spectral Element Method } \\ \text { TNR } & \text { Times New Roman } \\ \text { UCLA } & \text { University of California, Los Angeles } \\ \text { URANS } & \text { Unsteady Reynolds Averaged Navier Stokes }\end{array}$





\begin{abstract}
The project presents results of a computational fluid dynamics (CFD) model developed by the Oak Ridge National Laboratory team for a synthetic jet produced by an actuator. Numerical results were compared against experimental data provided by Actasys, Inc. Three CFD packages were used for this work that are Star-CCM+, Nek5000 and OpenFOAM. A sensitivity analysis was performed with Star-CCM+ and provided more insights on how to optimize the design of the actuator. Nek5000 was used to obtain a high-resolution solution of the synthetic jet. In a exploratory stage, a CFD model of the actuator was also developed in OpenFOAM. All CFD models were able to capture the main flow features.
\end{abstract}

\title{
1. OBJECTIVE AND BACKGROUND
}

Aerodynamic drag, or wind resistance, accounts for more than $50 \%$ of the fuel consumed by heavy trucks at highway speeds. The shape of these vehicles is determined by logistic requirements and cannot be readily changed for aerodynamic requirements. Active flow control (AFC) uses man-made jets of air to virtually reshape the vehicles without requiring actual changes to their shape (see Fig. 1). The performance of the synthetic jet is fundamental to the success of the technology. Previous approaches to AFC relied upon jets generated by air compressors. While this method proved that AFC improves aerodynamics, the energy consumption of the compressors led to an overall negative effect on system efficiency. In contrast, Actasys, Inc., uses a net zero mass flow actuator, based on a piezoelectric membrane in an open cavity, resulting in dramatically improved efficiency. Actasys has spent much of the past two years optimizing the performance of the jet actuators through an experimental process of design, build, test, and iterate.

Through this process, a number of critical variables in the design of the actuator have been identified. For each given membrane, optimum actuator design depends on up to eight variables with multiple outputs, resulting in an enormous test matrix and a tremendous challenge for the experimental approach.

Development of the actuators could be dramatically accelerated by computational fluid dynamics (CFD) modeling. The contractor, UT-Battelle, has assembled a multi-disciplinary team from Oak Ridge National

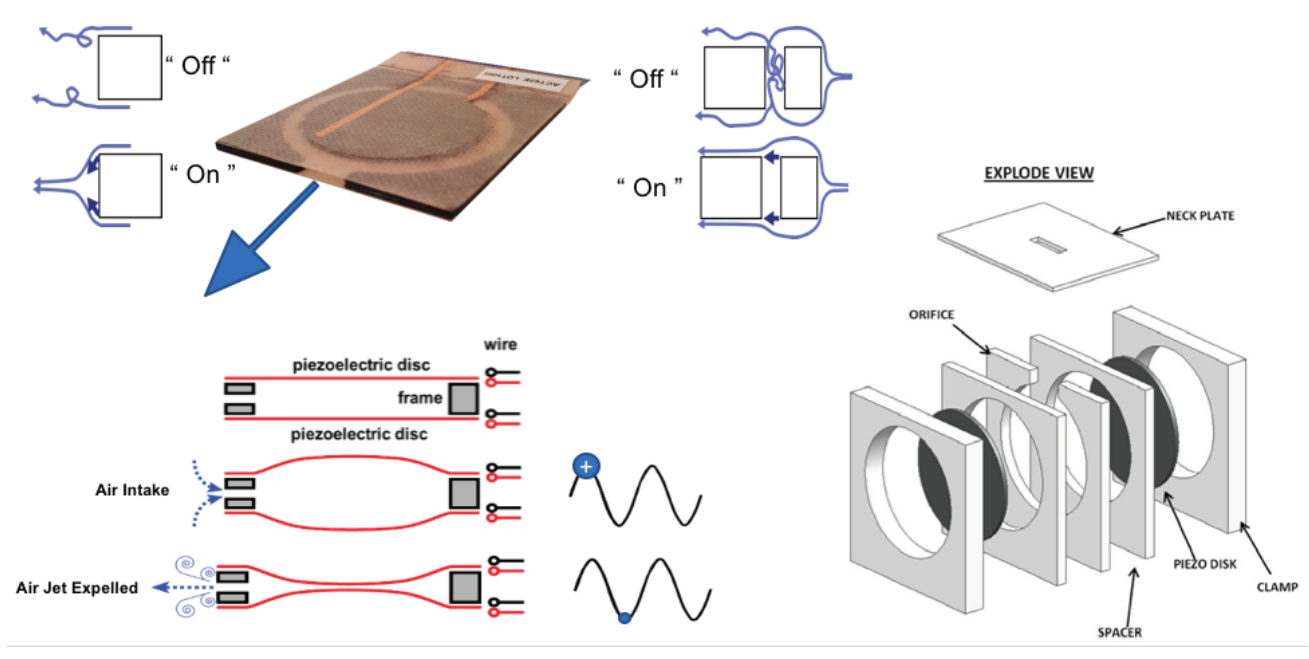

Figure 1. Sketch and concept of an actuator 
Laboratory (ORNL) with the expertise and capabilities to assist the participant in this effort. The contractor will develop a high-fidelity 3D CFD model and perform a multivariable parametric study of these variables to predicatively optimize the membrane and actuator design.

In the late 1990s and early 2000s, many studies of net zero flow actuators sought to establish experimentally calibrated reduced order models to describe actuator performance. More recent studies have focused on direct numerical simulation (DNS) of the actuator, nozzle and jet interaction. The current need, however, is to optimize both the actuator performance and the ability of the device to favorably impact surface boundary layers in turbulent flows. To do this, it is important for the transient flow structures generated both in the actuator and the interaction of the jet with the boundary layer to be well characterized. The contractor will evaluate the applicability of Nek5000, a highly scalable open source CFD code with dynamic mesh modification capabilities for optimization of the cavity and nozzle. The contractor will also determine whether the flow field can be effectively modeled using the more cost effective wall-resolved large eddy simulations (LES). Either would provide sufficient resolution to support Bayesian calibration of reduced order models for engineering analysis.

\section{BENEFITS TO THE DOE MISSION}

The work performed under this cooperative reserach and development agreement (CRADA) directly supported the US Department of Energy (DOE) mission in the Advanced Manufacturing Office of the Office of Energy Efficiency and Renewable Energy by providing computational support to Actasys Inc. to allow for faster deployment of their product - a synthetic jet actuator for active flow control on heavy duty vehicles. Actasys is working on a drag reduction system using synthetic jet actuators (SJAs) to improve energy efficiency of heavy duty vehicles (HDVs). Although the system has shown great potential for reducing fuel consumption, the Actasys "build-to-test" approach restricts the system optimization for manufacturing and commercialization. Collaboration with ORNL unlocks the nearly unlimited opportunities of additive manufacturing methods for fabrication of SJA devices, as well as their integration into HDV components. The high performance computing (HPC) -based computational fluid dynamics (CFD) tools were used to analyze the performance of the tractor-mounted SJA system. The work includes multivariable parametric studies, reduced order model strategy, and studies of potential compatibility with commercial CFD software platforms. The Actasys experimental database is used for validation. The successful commercialization of Actasys technology has the potential to save 2.5 Billion gallons of diesel fuel annually accross the entire US tractor-trailer fleet.

\section{TECHNICAL DISCUSSION OF WORK PERFORMED}

\subsection{SUMMARY OF PROJECT PHASES}

\subsection{Initial CFD Model Development}

The project team used high resolution computational fluid dynamics methods on HPC platforms to provide insight into the underlying physical phenomena that impact the performance of SJAs. Additive manufacturing methods provide opportunities for nearly unlimited optimization of harmonic cavity and nozzle designs for specific applications. Unsteady flow structures were evaluated along with the 
instabilities that appear in Actasys design. This evaluations were performed directly using high resolution methods to gain insights to enable the development of a reduced order modeling strategy to customize the design for its intended use. These devices could be applied to boundary layer modification for AFC as a demonstration of this capability. A preliminary intermediate resolution CFD model was constructed using the Unsteady Reynolds Averaged Navier Stokes (URANS) models of the commercial CFD code STAR-CCM+ to scope model sensitivities and resultant jet velocities profiles. The model uses a highly resolved mesh suitable for all Y+ number type turbulence models, and the simulation relies on the native mesh morphing capabilities of the code to move the oscillating wall boundaries. Simulation results for the nominal configuration, shown in Fig. 2, exhibit areas of significant acceleration near the short edges of the elongated rectangular slot, which are expected to contribute to the unique jet shape observed in experimental testing. The profile within the slot nozzle cannot be directly observed in the experiments. A high resolution model was constructed simultaneously, resolving all turbulence structures in a direct numerical simulation (DNS) using the high performance CFD code Nek5000. This model uses a computational mesh that is similar in characteristic size to the STAR-CCM+ model, but the spectral element simulation is executed at a spatial resolution that is on the order of 8 to 10 times higher resolution versus the previous STAR-CCM+ model, which was only on the order of 2 times higher in resolution. The native mesh morphing capabilities of the Nek5000 code were used to move and deform the wall boundary to close the actuator cavity and propel the jet through the nozzle. Scoping simulations in two and three dimensions were completed on internal ORNL production computing facilities, as shown in Fig. 3, in preparation for full-scale extended time domain simulations on HPC resources allocated to this project.

\subsection{Design Exploration Analyses}

Initial efforts focused on the development of CFD models of the Actasys synthetic jet actuator using unsteady Reynolds averaged Navier-Stokes (URANS) simulations that resolve the surface motion of the actuator's piezoelectric membrane. Results were validated against high-resolution particle image measurements of averaged jet velocities (over 500 snapshots) at two planes across the outlet of the SJA. Based on this benchmark, a highly optimized meshing strategy was implemented to reduce the run time while preserving predictive accuracy for quantities of interest, such as the peak time averaged velocity of the jet produced. The strategy was validated for a variety of additional conditions and geometries using available experimental data from Actasys. A commercial design search and optimization package, HEEDS, was integrated with the coarse resolution URANS model to facilitate more efficient exploration of the impacts of nozzle design options on the performance of the actuator. The model was updated so that selected input fields are parameterized and can be altered by the optimization package. The HEEDS package automatically updates the CFD model based on the selected state space sampling strategy and executes as many cases as necessary to satisfy the completion criteria. An initial sensitivity study was completed using this approach, demonstrating the impact of design parameters - membrane disk displacement, membrane disk oscillation frequency, and the length of the nozzle - on the peak time-averaged velocity of the jet produced. A sensitivity study was completed in which a few hundred cases run were to completion to demonstrate the capability, as shown in Fig. 4. A high-resolution reference model using a wall-resolved LES method was also developed to provide further insight into the physical phenomena defining actuator performance. Numerical solutions of the synthetic jet problem using the Nek5000 CFD code were run on ORNL HPC resources and are shown in Figures 1-3. The numerical solution obtained with Nek5000 is in good agreement with the experimental data. Some points of concerns include the predicted shape of the jet at the exit of the nozzle with Nek5000, which is thinner than observed 

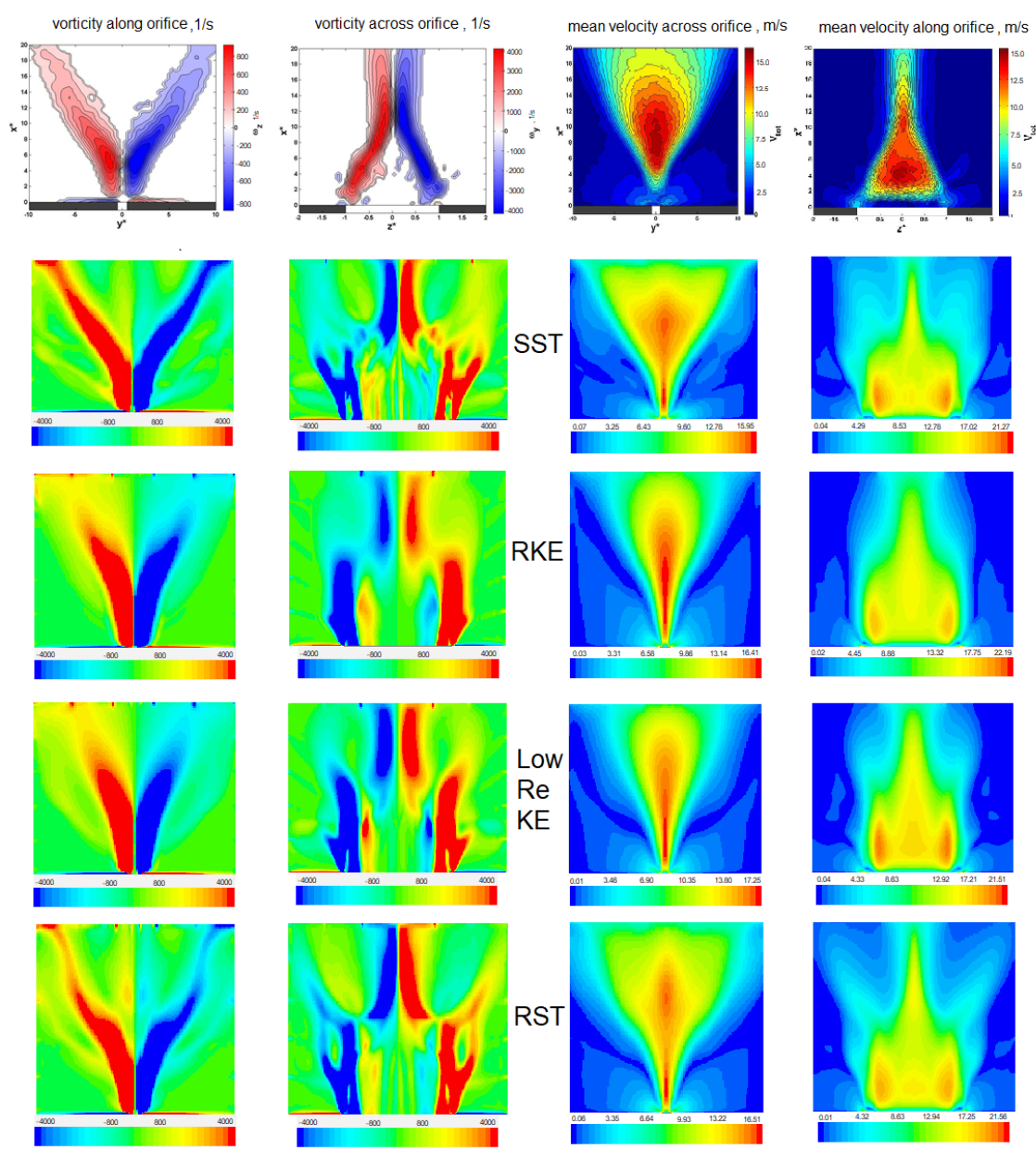

Figure 2. SJA simulation of disk motion and induced jet formation when operated at a frequency of $1,000 \mathrm{~Hz}$ showing the average orifice velocity and vorticity evolution in time for ten cycles of the actuator. The top images are experimental, and the remaining images evaluate different turbulence models (shear stress turbulence (SST), renormalized k-epsilon (RKE), low RKE, and Reynolds-stress turbulence (RST)

in the experimental data. Drag on each wall of the nozzle was also computed with Nek5000 as a function of time over one cycle. The drag is an example of data extracted from the high-fidelity CFD solution that can be used to benchmark the low-order model developed with OpenFOAM. A partnership has been established with a project in progress at the University of California, Los Angeles (UCLA) to evaluate the structural deformation of the membrane itself during the piezoelectric cycle. Data will be exchanged with that structural simulation team to better evaluate the effects of membrane deformation shapes. 

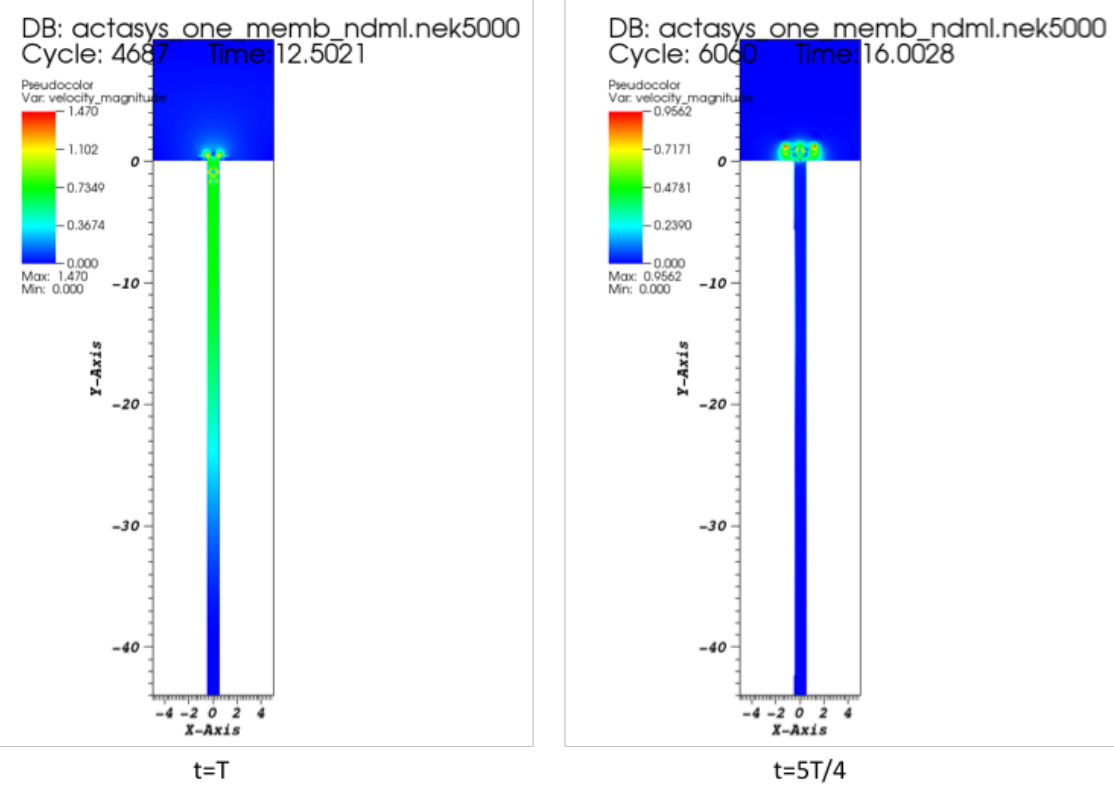

Figure 3. Snapshots of detailed velocity profiles across the narrow gap of a SJA operating at $1,000 \mathrm{~Hz}$, as predicted by high-resolution DNS. Time $t=T$ aligns with the completion of the compressive stroke

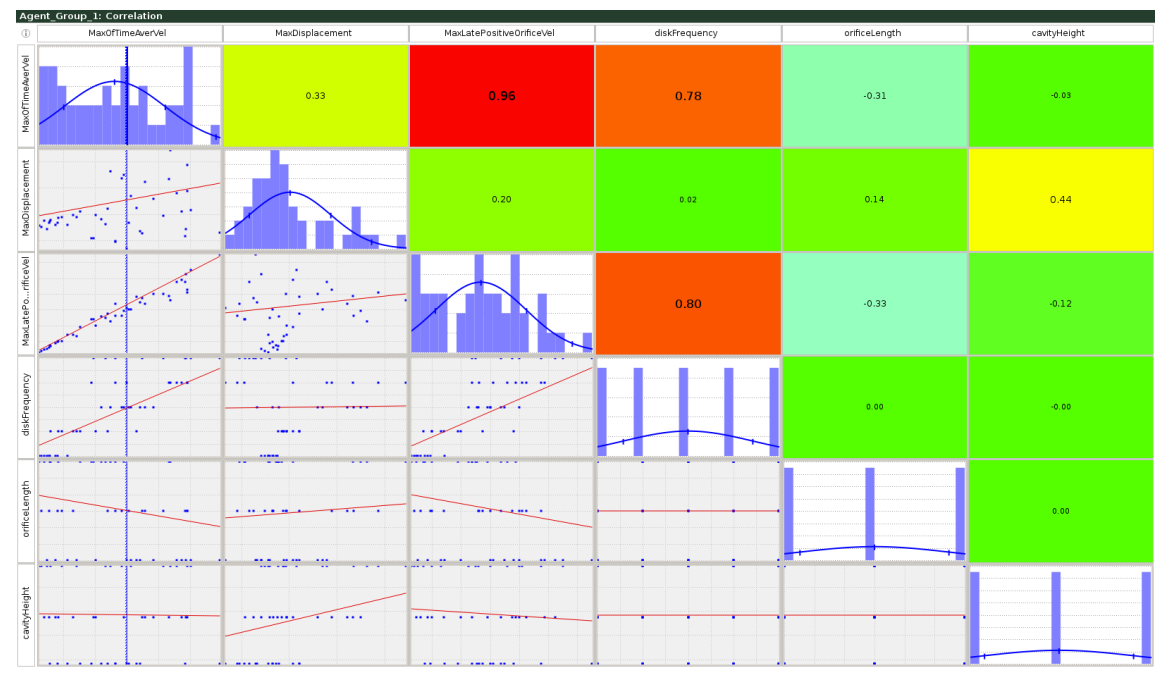

Figure 4. Demonstration sensitivity study evaluating impact of simple design changes on the maximum time-averaged velocity of the jet. As should be expected, the jet velocity is strongly correlated to the displacement of the membrane that drives the air flow.

\subsection{Practical Implementation, Model calibration to Displacement Data and Preliminary drag Evaluations}

The flow simulations require an assumption for the disk displacement. The assumption is used as a boundary condition for deforming the computational grid. In the initial experimental data, only the 
maximum displacement was measured. In order to define the complete disk motion, it was assumed that the disk deforms following a sine function. Recently, analyzing the obtained flow results and comparing them to available data, some deviations in the orifice velocity were discovered. Also, in the design exploration studies, one of the parameterization factors is the driving frequency and its dependence on the displacement. When a frequency sweep is performed, one of the key questions is whether the sine assumption is valid throughout the entire frequency range. Experimental work was initiated to answer these questions. Detailed, two-dimensional profiles of the disk displacement were produced. Then these profiles were sampled along the main directions in the disk center. Plots are given in Fig. 5. They confirm the sine assumption up to frequencies of about $800 \mathrm{~Hz}$. Frequencies in that range $(200-800 \mathrm{~Hz})$ enclose the first resonance peak. This peak typically results from the disk's natural frequency. At driving frequencies of about $800 \mathrm{~Hz}$ and above, the disk shape begins to deviate from sine law. A second mode starts to appear, and the sine assumption is invalid for the second resonance peak. This finding is very important, because the initial analyses focused on the second peak, which is larger. The predicted jet velocities, which were higher than those measured, may be due to the improperly assumed disk deformation shape. Experiments continued to introduce another design parameter: i.e. the cavity height (depth). Two additional freight type actuators were fabricated and tested in a frequency sweep mode to define the displacement shape and amplitude. Then a cross-correlation function was derived to implement the double parameterization logic in the STAR-CCM+ design evaluation algorithm. This work completed the design exploration analyses using the previously developed computational models. The calculation automation and statistical processing of results was performed using HEEDS software and implemented in STAR-CCM+.

OpenFOAM CFD code was also used to model a synthetic jet produced by Actasys actuator. Numerical solutions obtained with the URANS Realizable k-epsilon incompressible turbulent model are shown in Fig. 6; the image on the left shows the time-averaged velocity over 6 full cycles. Preliminary numerical results show that OpenFOAM can capture the main flow features. In a second phase, an OpenFOAM model of a turbulent flow around a car was developed and tested. Numerical results obtained with an incompressible model and the k-omega SST URANS model are shown in Fig. 6, right. This exploratory work is preliminary to modeling a full-scale vehicle (car or truck) with actuators to quantify their effect on the drag.

\subsection{COMPUTATIONAL METHOD}

The non-dimensional forms of the incompressible Navier-Stokes equations are solved with the highly efficient and massively parallel spectral element solver Nek5000 [2]. In the spectral element method (SEM), the weak forms of the equations are discretized in space by the Galerkin approximation. The solution is expanded into the $\mathrm{n}^{\text {th }}$ order tensor product Lagrange polynomials based on the Gauss-Lobbato-Legendre (GLL) quadrature points within hexahedral conforming elements [1]. Time integration of the continuity and momentum equations is achieved with a semi-implicit scheme in which the viscous terms of the Navier-Stokes equations are treated implicitly using third-order backward differentiation (BDF3). The non-linear terms are treated explicitly with a third-order scheme (EXT3). SEM offers the flexibility to accommodate complex geometries and the high-order accuracy required to solve turbulent flows. It also exhibits little numerical dissipation and dispersion, which greatly matters when solving high Reynolds flow and integrating over long period of time [1]. Nek5000 also implements the Arbitrary Lagrangian/Eulerian (ALE) approach to model mesh deformation from moving boundaries. This formulation was originally developed by Ho et al. [3] and later extended to the so-called Pn-Pn formulation 

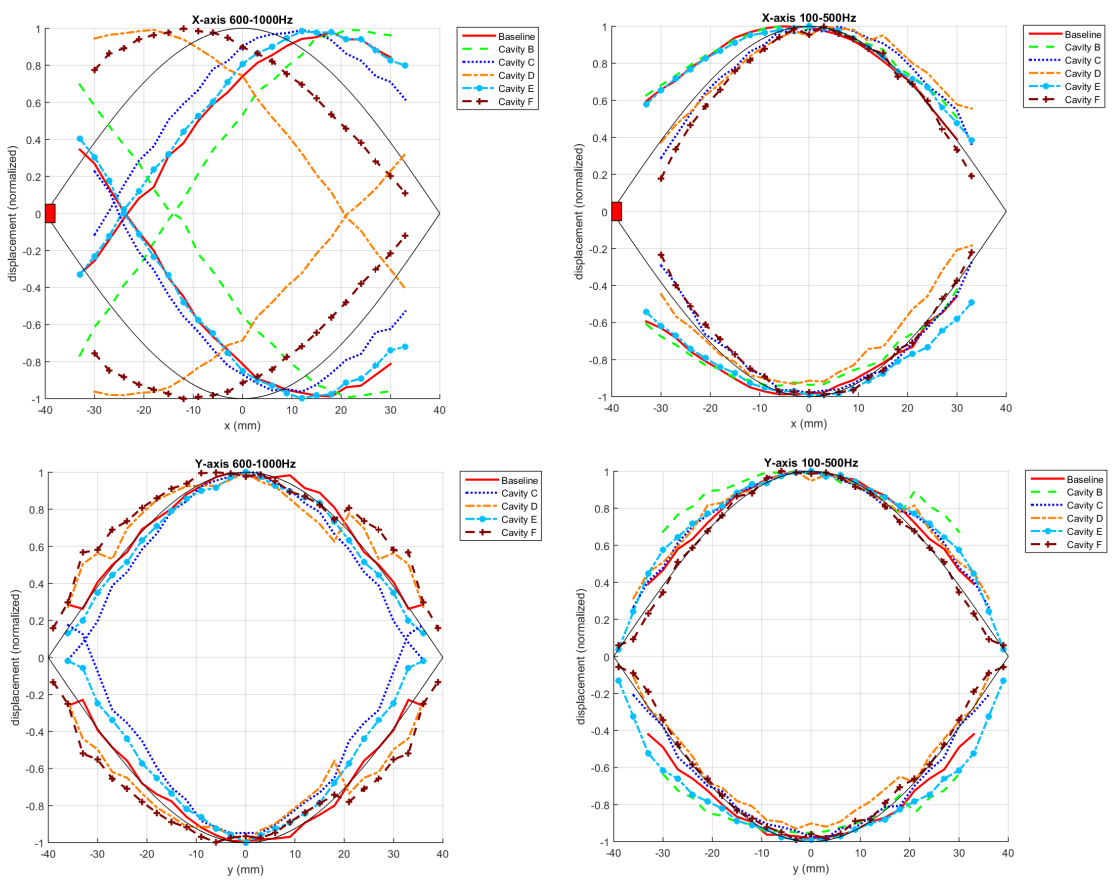

Figure 5. Disk shapes in the function of the driving frequency for low and high ranges. Comparison to a sine-shaped profile is provided, and the resultant difference in the displaced volume is calculated.
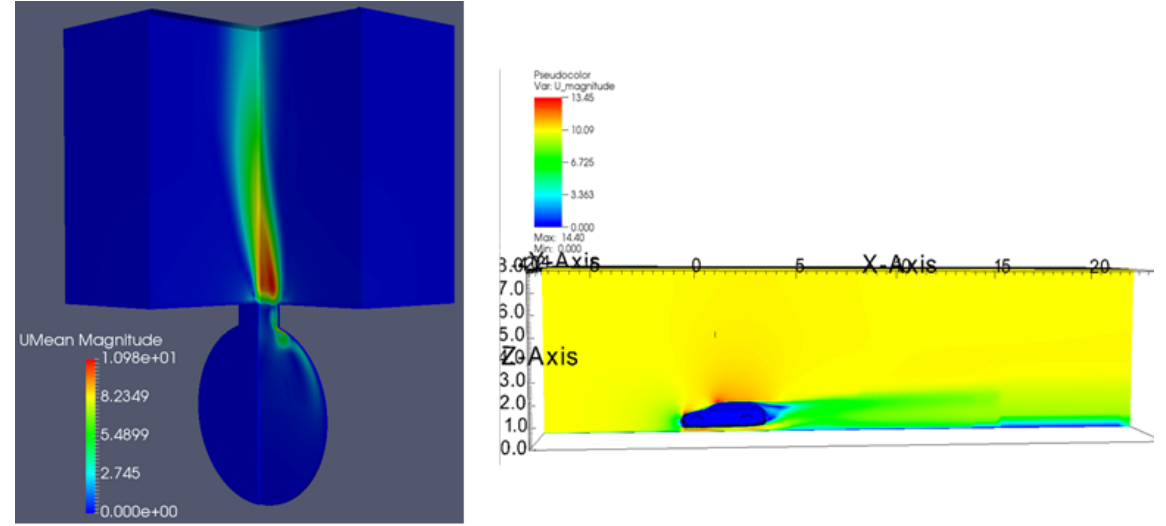

Figure 6. Clipped view of an SJA. Numerical solution obtained with OpenFOAM.3 (left). Slice view of the 3-D turbulent flow around a car (right)

in [4]. The ALE implementation is used in this work to model the deformation of the membrane in the cavity of the actuator. 


\subsection{NON-DIMENSIONALIZATION OF THE INCOMPRESSIBLE NAVIER-STOKES EQUATIONS FOR A SYNTHETIC JET}

Solving for the non-dimensional form of the Navier-Stockes equations presents valuable advantages, as it minimizes round-off errors, offers physical insights, and allows for assessment of the relative importance of terms in the model equations. For the problem of interest, the non-dimensional Navier-Stokes equations are solved with a moving boundary condition to model the membrane motion inside the cavity. It is assumed that the fluid does not affect the membrane shape. This section details the steps that were taken to non-dimensionalize the Navier-Stokes equations and the moving boundary conditions in a consistent manner for both 2-D and 3-D computational models. Section 3.6.1 introduces the reader to the problematic of reference velocity for a synthetic jet. The logic followed to derive the synthetic jet reference velocity is detailed in Section 3.6.2. The final non-dimensionalized Navier-Stokes equations are presented in Section 3.6.5.

\subsubsection{Non-dimensionalization of the incompressible Navier-Stokes equations with moving boundary}

This section introduces the mathematical notations and equations that will be used to derive the non-dimensionalized Navier-Stokes equations and the set of boundary conditions to close the system. The 3D incompressible Navier-Stokes equations (INS) are written in a function of the fluid velocity vector $\vec{u}$, as well as the density $\rho$ and the viscosity $\mu$ that are constant fluid properties. The INS equations are recalled below in Eq. (1):

$$
\begin{gathered}
\nabla \cdot \vec{u}=0 \\
\rho\left(\partial_{t} \vec{u}+\nabla \cdot(\vec{u} \otimes \vec{u})\right)+\nabla P=\mu \Delta \vec{u}
\end{gathered}
$$

An equation for the moving boundary condition function of time and space is also considered. For the purpose of this report, the membrane is assumed to be initially located at $\vec{x}_{0}$. During the transient, the membrane vibrates with a frequency $f$, and achieves a maximum displacement $D$. The displacement $d(\vec{x}, t)$ is assumed to be a function of the membrane shape $A_{\text {shape }}(\vec{x})$ and a sine law for temporal variations, as follows:

$$
d(\vec{x}, t)=\vec{x}_{0}+D A_{\text {shape }}(\vec{x}) \sin (2 \pi f t),
$$

where the variable $t$ denotes time.

To non-dimensionalize the INS equations, reference or characteristic quantities must be chosen. Thus, $t_{0}$, $U_{0}$ and $L_{0}$ denote the characteristic time, the characteristic velocity, and the characteristic length, respectively. Since the density and the viscosity remain constant during the transient, $\rho_{0}=\rho$ and $\mu_{0}=\mu$ are chosen. Following these assumptions, a reference quantity for the pressure can be derived and defined as $P_{0}=\rho_{0} U_{0}^{2}$. Using the characteristic length, we defined nondimensional variables can be defined with respect to the dimensional variables now denoted by $\hat{\bullet}$ :

$$
\overrightarrow{\hat{u}}=U_{0} \vec{u}, \hat{P}=\rho U_{0}^{2} P, \overrightarrow{\hat{x}}=L_{0} \vec{x} \text { and } \hat{t}=t_{0} t=\frac{L_{0}}{U_{0}} t .
$$

Note that the notations have been changed to simplify the remainder of the derivation. The INS equations can now be expressed as a function of the non-dimensional variables and the Reynolds number $R e_{0}$; Eq. (3) 
and Eq. (1) yield:

$$
\begin{gathered}
\frac{U_{0}}{L_{0}^{2}} \nabla \cdot \vec{u}=0 \\
\partial_{t} \vec{u}+\nabla \cdot(\vec{u} \otimes \vec{u})+\nabla P=\frac{1}{\operatorname{Re}_{0}} \Delta \vec{u}, \text { with } \operatorname{Re}_{0}=\frac{\rho_{0} U_{0} L_{0}}{\mu_{0}}
\end{gathered}
$$

The non-dimensional continuity equation Eq. (4a) can be further simplified as the ratio $\frac{U_{0}}{L_{0}^{2}}$ cannot be physically equal to zero if a flow exists:

$$
\frac{U_{0}}{L_{0}^{2}} \nabla \cdot \vec{u}=0 \rightarrow \nabla \cdot \vec{u}=0
$$

The incompressible continuity equation has its final form and will no longer be carried out in the remaining derivations of this section. The displacement of the membrane given in Eq. (2) is also non-dimensionalized using Eq. (3) which yields:

$$
\begin{gathered}
L_{0} d(\vec{x}, t)=L_{0} \vec{x}_{0}+L_{0} D A_{\text {shape }}(\vec{x}) \sin \left(2 \pi f t_{0} t\right), \\
d(\vec{x}, t)=\vec{x}_{0}+D A_{\text {shape }} \sin \left(2 \pi \mathrm{St}_{0} t\right) \text { where } \mathrm{St}_{0}=\frac{f L_{0}}{U_{0}}
\end{gathered}
$$

where $\mathrm{St}_{0}$ is the Strouhal number.

Due to the nature of the problem under consideration (i.e., a flow triggered by a moving membrane treated as a moving boundary), the definition of the reference velocity is not obvious. In the next section, a method for deriving a reference velocity is presented.

\subsubsection{Reference velocity for an actuator}

When simulating or collecting experimental data of a synthetic jet produced by an actuator, a reference velocity is not readily available. The fluid velocity is not known in the nozzle. Furthermore, it is not set by a pump or by a Dirichlet boundary condition when dealing with experiments and simulations, respectively. The membrane velocity cannot be used as a reference velocity, since it cannot be measured with sufficient accuracy due to the high velocities involved. One way to derive a reference velocity is to perform a conservation of mass flow between the cavity where the membrane vibrates, and the nozzle; this is done over a half cycle. (Herein, a cycle corresponds to a period).

In this report and without lack of generality, a nozzle is assumed to be oriented in the z-direction with a cross section $A_{n o z z l e}$ in the x-y plane, and connected to a cavity below and containing only one membrane. Initially, the membrane is not deformed and is located $\vec{x}_{0}$. The displacement of the membrane over time is denoted by $d(\vec{x}, t)$, and described by Eq. (2). where $f$ is the frequency at which the membrane vibrates at, $D$ is the maximum displacement of the membrane, and $A_{\text {shape }}(\vec{x})$ is a normalized spatial function that describes the shape of the membrane (normalized function refers herein to a function that varies between 0 and 1).

When the membrane vibrates with a frequency $f$, the volume of the cavity $V(t)$ changes as a function of time $t$. Because of the change in the cavity volume, the fluid is forced to enter and exit the cavity through 
the nozzle. The fluid velocity in the nozzle is a function of space and time. Therefore, $\bar{U}(t)$ denotes the spatial-average fluid velocity at the top of the nozzle. Using the conservation of mass between the exit of the nozzle and the cavity, a relationship can be derived between the rate of variation of cavity volume $d V / d t$, the fluid velocity $\bar{U}(t)$, and the geometric parameters of the nozzle. Within a time $\Delta t$, the volume of air moved in the cavity is:

$$
\Delta m_{\text {air }}=\rho_{\text {air }}[V(t)-V(t+\Delta t)]
$$

Within the same time $\Delta t$, the amount of air that exits or enters the nozzle is:

$$
\Delta m_{\text {air }}=\rho_{\text {air }} \bar{U}(t) A_{\text {nozzle }} \Delta t
$$

which yields the following relationship when assuming an incompressible flow:

$$
V(t)-V(t+\Delta t)=\rho_{\text {air }} \bar{U}(t) A_{\text {nozzle }} \Delta t .
$$

Taking the limit $\lim \Delta t \rightarrow 0$, the rate of variation of cavity volume is obtained:

$$
\frac{d V}{d t}=\bar{U}(t) A_{\text {nozzle }}
$$

The relationship given in Eq. (10) requires an expression for the volume of the cavity as a function of time to compute the fluid velocity $\bar{U}(t)$ achieved at the exit of the nozzle. The volume of the cavity can be derived by integrating the displacement given in Eq. (2), but this requires further assumptions, notably an expression for $A_{\text {shape }}(\vec{x})$. It is proposed in Section 3.6.3 and in Section 3.6.4 to investigate 2D and 3D models of an actuator, respectively, and to derive an expression for the maximum velocity achieved in the nozzle for each case.

$$
u(x, y, z, t)=U_{p} A(x, y, z) \sin (2 \pi f t),
$$

where $U_{p}$ is the peak velocity achieved in the nozzle, and $A(x, y, z)$ is a normalized function. The velocity variations with respect to time are assumed periodic of the same frequency as the membrane frequency $f$. The spatial-average velocity is denoted by $\hat{u}(t)$ and is computed by integrating the spatially dependent terms in Eq. (11), over the nozzle volume $V_{\text {nozzle }}$ :

$$
\hat{u}(t)=\hat{U} \sin (2 \pi f t) \text { where } \hat{U}=\frac{1}{V_{\text {nozzle }}} \int_{V_{\text {nozzle }}} U_{p} A(x, y, z) d V
$$

The variable $\hat{U}$ is the maximum velocity of the spatial-averaged velocity in time.

The time-averaged and spatial-averaged velocity are obtained by integrating Eq. (12) over a half-cyle, i.e., $T / 2$ :

$$
\bar{U}=\frac{1}{T / 2} \int_{0}^{T / 2} \hat{u}(t) d t=\frac{2}{\pi} \hat{U}
$$

In the remainder of this report, the notations are changed; $U_{\max }=\hat{U}$ is referred to as the maximum velocity, and $U_{a v g}=\bar{U}$ is referred to as the average velocity. 


\subsubsection{Derivation of a reference velocity for a 2-D model of an actuator}

For the purpose of this section and without lack of generality, it is assumed that the membrane is of length $L$, is vertically oriented along the $y$-axis, and its displacement is a function of time and space on the same model as Eq. (2):

$$
d(y)=\frac{h_{0}}{2}+D A_{n}(y) \sin (2 \pi f t),
$$

where $h_{0}$ is the width of the nozzle and the initial width of the cavity, $D$ is the maximum displacement, $A_{n}(y)$ is the normalized local displacement, $f$ is the membrane frequency, and $t$ is time. The volume (surface in a 2D model) of the cavity is obtained by integrating Eq. (14) over the cavity's height, which yields:

$$
V(t)=\int_{0}^{L} d(y) d y=\frac{L h_{0}}{2}+D \sin (2 \pi f t) \int_{0}^{L} A_{n}(y) d y
$$

To go further in the derivation, one must assume an expression for the normalized local displacement $A_{n}(y)$. Two displacement shapes are considered, namely, a parabolic displacement $A_{n}^{p}$, and a sine-law displacement $A_{n}^{s}$, with the following expression:

$$
A_{n}^{p}(y)=\frac{y(y-L)}{A_{n, \max }} \text { where } A_{n, \max }=\frac{L^{2}}{4},
$$

and

$$
A_{n}^{s}(y)=\sin \left(\pi \frac{y}{L}\right)
$$

The corresponding volume displaced for parabolic and sine-law displacement shapes proposed in Eq. (16a) and Eq. (16b), respectively, are:

$$
V^{p}(t)=\frac{L h_{0}}{2}+\frac{2}{3} D L \sin (2 \pi f t)
$$

and

$$
V^{s}(t)=\frac{L h_{0}}{2}+\frac{2}{\pi} D L \sin (2 \pi f t)
$$

which can be recast under the following generic form:

$$
V^{i}(t)=\frac{L h_{0}}{2}+\alpha_{i}^{2 D} D L \sin (2 \pi f t) \text { with } \alpha_{p}^{2 D}=\frac{2}{3} \text { and } \alpha_{s}^{2 D}=\frac{2}{\pi} .
$$

Substituting the results from Eq. (18) for the volume displaced into Eq. (10), it yields an expression linking the membrane frequency $f$, the geometric parameters of the nozzle $\left(D, L\right.$ and $\left.h_{0}\right)$, and the spatial-average velocity in time at the top of the nozzle $\bar{U}(t)$ :

$$
\frac{d V^{i}(t)}{d t}=2 \pi f \alpha_{i}^{2 D} D L \cos (2 \pi f t)=\bar{U}(t) h_{0} \rightarrow \bar{U}(t)=\frac{2 \pi f \alpha_{i}^{2 D} D L \cos (2 \pi f t)}{h_{0}}
$$

From Eq. (19), it is understood that the spatial average velocity in time $\bar{U}(t)$, follows a sine-law with the same frequency as the membrane frequency, and that the maximum velocity is reached when the cosine function reaches extrema, that is, $\cos (x)= \pm 1$, which corresponds to $t=\frac{k}{f}$ where $k$ is an even integer. The maximum spatially averaged velocity, or the maximum velocity, in time is:

$$
\bar{U}_{\max }=\frac{2 \pi f \alpha_{i}^{2 D} D L}{h_{0}}
$$


The time-average velocity $\bar{U}_{\text {avg }}$ can also be related to the maximum velocity $\bar{U}_{\text {max }}$, by integrating Eq. (19) over a half-cycle $[-T / 4, T / 4]$ :

$$
\bar{U}_{\text {avg }}=\frac{1}{T / 2} \int_{-T / 4}^{T / 4} \bar{U}(t) d t=\frac{2}{\pi} \bar{U}_{\max }
$$

Remark 1 When integrating the velocity $\bar{U}(t)$ to compute the time-average velocity $\bar{U}_{\text {avg, }}$ the time internal that corresponds to a half cycle must be chosen so that the membrane velocity remains of the same sign: $[-T / 4, T / 4]$. When choosing a different half-cycle interval in which the membrane reaches one extrema and thus changes sign, the average velocity is obtained by integrating the absolute value of $\bar{U}(t)$.

Now a numerical application relevant to the problem of interest can be performed using data provided in Table 1 to compute the average fluid velocity $\bar{U}_{\text {avg }}$ and the maximum fluid velocity $\bar{U}_{\text {max }}$ in the nozzle, the Reynolds number, and the Strouhal number. The working fluid is air, the physical properties of which are taken at atmospheric conditions.

\begin{tabular}{|c|c|c|c|c|c|}
\hline $\mathrm{h}(\mathrm{mm})$ & $\mathrm{L}(\mathrm{mm})$ & $\mathrm{D}(\mathrm{mm})$ & $\mathrm{f}(\mathrm{Hz})$ & $\rho_{\text {air }}\left(\mathrm{kg} / \mathrm{m}^{3}\right)$ & $\mu_{\text {air }}(\mathrm{kg} /(\mathrm{m} \cdot \mathrm{s})$ \\
\hline 1.0 & 40.0 & $8 \times 10^{-5}$ & 1125 & 1.16 & $1.855 \times 10^{-5}$ \\
\hline
\end{tabular}

Table 1. Geometric data for a 2-D actuator.

The nozzle width is chosen as the characteristic length, i.e., $L_{0}=h_{0}$. The average and maximum velocities can be chosen as the reference velocity $U_{0}$; at this stage, it is still unclear which one would be more appropriate to correctly non-dimensionalize the problem. Therefore, it is proposed to compute the Reynolds number and the Strouhal numbers from either velocities. Results are summarized in Table 2.

\begin{tabular}{|c|c|c|c|c|c|c|}
\hline shape & $U_{\text {avg }}(\mathrm{m} / \mathrm{s})(\mathrm{Eq} .(20))$ & $U_{\max }(\mathrm{m} / \mathrm{s})(\mathrm{Eq} .(21))$ & $\operatorname{Re}_{0}\left(U_{\text {avg }}\right)$ & $\mathrm{St}_{0}\left(U_{\text {avg }}\right)$ & $\operatorname{Re}_{0}\left(U_{\max }\right)$ & $\mathrm{St}_{0}\left(U_{\max }\right)$ \\
\hline parabolic & 9.60 & 15.08 & 600 & 0.117 & 943 & 0.075 \\
\hline sine-law & 9.17 & 14.40 & 573 & 0.123 & 900 & 0.078 \\
\hline
\end{tabular}

Table 2. Non-dimensional numbers for a 2-D actuator.

When defining the reference velocity with respect to the average velocity $\bar{U}_{a v g}$, the Reynolds number and Strouhal numbers are closer to the experimental data: $\mathrm{Re}_{\text {exp }}=615$ and $\mathrm{St}_{\text {exp }}=0.115$. It is also noted that the values of the Reynolds and Strouhal numbers depend in part on the deformation model used for the membrane when it vibrates, i.e., parabolic versus sine-law. Between a parabolic deformation and a sine-law deformation, the Reynolds number and the Strouhal number varies by $5 \%$.

Remark 2 For the case in which the average velocity is defined as the maximum velocity divided by $\pi$, which corresponds to the definition chosen by Actasys, the Reynolds numbers given in Table 2 are to be divided by $\pi$, while the Strouhal numbers are to be multiplied by $\pi$.

Remark 3 When considering a cavity with two membranes instead of one as assumed in this report, the displaced volume is twice as much, which means that $V$ should be substituted with $2 V$ in Eq. (10). The average and maximum velocities will then be multiplied by two in Table 2. 


\subsubsection{Derivation of a reference velocity for a 3-D model of an actuator}

In a 3D model of a synthetic jet actuator, the cylindric cavity still contains one membrane of a circular shape and a radius $R=20$ millimeter that vibrates at a frequency $f=1125 \mathrm{~Hz}$. The membrane is initially at rest and parallel to the $\mathrm{x}-\mathrm{y}$ plan. The cavity is connected to a nozzle of rectangular cross section $A_{\text {nozzle }}$. The membrane displacement $d(\vec{x}, t)$ is assumed to follow Eq. (2). The same step-by-step process tjat was implemented in Section 3.6.3 is followed: (1) the volume of air displaced is computed when assuming either a parabolic displacement shape (paraboloid) or a sine-law displacement shape, (2) an expressions for the maximum and average velocities are then derived using Eq. (10), and (3) a numerical application is performed using geometric parameters and properties of air at atmospheric conditions.

Two expressions are considered for the normalized displacement $A_{\text {shape }}$, namely, a paraboloid displacement $A_{\text {shape }}^{p}$, and a sine-law displacement $A_{\text {shape }}^{s}$, whose expressions are given below:

$$
\begin{gathered}
A_{\text {shape }}^{p}(y, z)=\frac{h}{R^{2}}\left(y^{2}+z^{2}\right), \\
A_{\text {shape }}^{s}(y, z)=\cos \left(\frac{\pi r}{2 R}\right) \text { where } r^{2}=y^{2}+z^{2} .
\end{gathered}
$$

The corresponding volumes of air displaced by the membrane for the paraboloid and the sine-law displacements are:

$$
\begin{gathered}
V_{n}^{p}=V_{0}+\frac{\pi}{2} D R^{2} \sin (2 \pi f t), \\
V_{n}^{s}=V_{0}+\frac{4(\pi-2)}{\pi} D R^{2} \sin (2 \pi f t),
\end{gathered}
$$

where $V_{0}$ is the initial volume of the cavity. The expressions of the volumes for the paraboloid and sine-law displacements can be recast under the generic form:

$$
V^{i}(t)=V_{0}+\alpha_{i}^{3 D} D R^{2} \sin (2 \pi f t) \text { with } \alpha_{p}^{3 D}=\frac{\pi}{2} \text { and } \alpha_{s}^{3 D}=\frac{4(\pi-2)}{\pi} .
$$

Substituting the results from Eq. (24) for the volume displaced into Eq. (10) it yields an expression linking the membrane frequency $f$, the geometric parameters of the nozzle and the cavity $\left(D, R\right.$ and $\left.A_{n o z z l e}\right)$, and the spatial-average velocity in time at the top of the nozzle $\bar{U}(t)$ :

$$
\frac{d V^{i}(t)}{d t}=2 \pi f \alpha_{i}^{3 D} D R^{2} \cos (2 \pi f t)=\bar{U}(t) A_{\text {nozzle }} \rightarrow \bar{U}(t)=\frac{2 \pi f \alpha_{i}^{3 D} D R^{2} \cos (2 \pi f t)}{A_{\text {nozzle }}}
$$

As in the two-dimensional case, the maximum velocity $U_{\text {max }}(t)$ is achieved when the time-dependent cosine is equal to one, which yields:

$$
\bar{U}_{\text {max }}^{i}=\frac{2 \pi f \alpha_{i}^{3 D} D R^{2}}{A_{\text {nozzle }}} .
$$

The time-average velocity $\bar{U}_{\text {avg }}$ is computed from Eq. (21) and Eq. (26).

A numerical application for a 3D actuator can now be performed using the geometric parameters and the fluid properties given in Table 3 . 


\begin{tabular}{|c|c|c|c|c|c|}
\hline$A_{\text {nozzle }}\left(\mathrm{mm}^{2}\right)$ & $\mathrm{R}(\mathrm{mm})$ & $\mathrm{D}(\mathrm{mm})$ & $\mathrm{f}(\mathrm{Hz})$ & $\rho_{\text {air }}\left(\mathrm{kg} / \mathrm{m}^{3}\right)$ & $\mu_{\text {air }}(\mathrm{kg} /(\mathrm{m} \cdot \mathrm{s})$ \\
\hline $1.2 \times 10^{-5}$ & 20.0 & $8 \times 10^{-5}$ & 1125 & 1.16 & $1.855 \times 10^{-5}$ \\
\hline
\end{tabular}

Table 3. Geometric data for a 2-D actuator.

Using the geometric parameters and air properties given in Table 3, along with the expressions for the average velocity (Eq. (21)) and the maximum velocity (Eq. (26)), the Reynolds number and Strouhal number are computed and summarized in Table 4 for the $3 \mathrm{D}$ actuactor.

\begin{tabular}{|c|c|c|c|c|c|c|}
\hline shape & $U_{\text {avg }}(\mathrm{m} / \mathrm{s})($ Eq. $(21))$ & $U_{\max }(\mathrm{m} / \mathrm{s})($ Eq. $(26))$ & $\operatorname{Re}_{0}\left(U_{\text {avg }}\right)$ & $\mathrm{St}_{0}\left(U_{\text {avg }}\right)$ & $\operatorname{Re}_{0}\left(U_{\max }\right)$ & $\mathrm{St}_{0}\left(U_{\max }\right)$ \\
\hline paraboloid & 18.85 & 29.61 & 1178 & 0.059 & 1850 & 0.038 \\
\hline sine-law & 17.44 & 27.39 & 1090 & 0.065 & 1712 & 0.041 \\
\hline
\end{tabular}

Table 4. Nondimensional numbers for a 3-D actuator.

The Reynolds and Strouhal numbers computed by Acatays are based on an average velocity $U_{\text {avg }}^{\text {Act }}$ that is defined as the maximum velocity divided by $\pi$. Under this assumption, the Reynolds number corresponding to $U_{\text {avg }}^{A c t}$ is obtained by dividing $\operatorname{Re}_{0}\left(U_{\max }\right)$ from Table 4 with $\pi$. Equivalently, the Strouhal number defined with respect to $U_{\text {avg }}^{\text {Act }}$ is obtained by multiplying $\operatorname{St}_{0}\left(U_{\max }\right)$ from Table 4 with $\pi$. The computed values are then:

$$
\operatorname{Re}_{0}\left(U_{\text {avg }}^{A c t}\right)=588 \text { and } \operatorname{St}_{0}\left(U_{\text {avg }}^{A c t}\right)=0.119
$$

which are to be compared against the experimental values, i.e., $R e_{\text {exp }}=615$ and $S t_{\text {exp }}=0.115$.

\subsubsection{Non-dimensionalized Navier-Stokes equations with moving boundary conditions}

At this stage it us useful to recall the system of nondimensional equations solved for simulating the flow produced by the actuator, along with the definitions of the nondimensional numbers. The nondimensional incompressible Navier-Stokes equations are given below as a function of the Reynolds number $\mathrm{Re}_{0}$ and the Strouhal number $\mathrm{St}_{0}$ :

$$
\left\{\begin{array}{l}
\text { Continuity equation: } \\
\nabla \cdot \vec{u}=0 \\
\text { Momentum equation: } \\
\partial_{t} \vec{u}+\nabla \cdot(\vec{u} \otimes \vec{u})+\nabla P=\frac{1}{\operatorname{Re}_{0}} \Delta \vec{u} \\
\text { Moving boundary condition: } \\
u_{d}(\vec{x}, t)=\mathrm{St}_{0} \cdot D \cdot A_{\text {shape }} \sin \left(2 \pi \mathrm{St}_{0} t\right)
\end{array}\right.
$$


The Reynolds and Strouhal numbers are computed from the geometric parameters of the cavity and the membrane frequency using the following relations:

$$
\left\{\begin{array}{l}
\mathrm{Re}_{0}=\frac{\rho_{0} U_{0} L_{0}}{\mu_{0}} \\
\mathrm{St}_{0}=\frac{f L_{0}}{U_{0}} \\
U_{0}=\frac{2 \pi f \alpha_{i}^{3 D} D R^{2}}{A_{\text {nozzle }}},
\end{array}\right.
$$

where all variables were previously defined in Section 3.6.3 and Section 3.6.4, and reference or characteristic variables are denoted by the subscript 0 .

A closer look at the definition of the Reynolds and Strouhal numbers indicates that the Reynolds number is a function of both geometric parameters (nozzle width, height and length, radius membrane) and the membrane parameters that are the frequency $f$ and the maximum displacement $D$. This implies that a change in frequency or in the geometric parameters will affect the Reynolds number. On other hand, the Strouhal number does not explicitly depend on the frequency; it only depends on geometric parameters such as maximum deformation, nozzle width, length and height, and membrane radius. Note that the maximum deformation is a strong function of the frequency, and actuators are designed to operate at the resonance frequency where the maximum displacement is larger. Furthermore, the period $T=1 / f$ is the Strouhal number in the nondimensional space. As the equations solved by Nek5000 are nondimensionalized, time-averaging operations will occur over a nondimensional period of time $\mathrm{St}_{0}$.

\subsubsection{Relationship between the maximum displacement and the membrane frequency}

The maximum displacement $D$ is a function of the membrane frequency $f$. To fully close the computational model and defined the boundary condition for the moving boundary that models the membrane, a relationship between the maximum displacement and the membrane frequency from experimental data had to be defined. A polynomial fit was derived from experimental data for the actuator under consideration for frequencies ranging from $200-800 \mathrm{~Hz}$. Coefficients of the polynomial fit are provided in Table 7 and plotted in Fig. 8. Note that the maximum displacement is normalized with respect to the nozzle height $h_{0}$. This polynomial fit is used in the remainder of this report to compute the maximum displacement based on the membrane frequency.

\subsection{SIMULATION SETUP}

This section describes the geometry and computational grid, and it also provides information on initial conditions, boundary conditions and averaging processes that occurred at run time.

\subsubsection{Geometry and computational grid}

In the present study, the geometry consists of two regions: the actuator and the outside box. The actuator is further divided into a cavity region and a nozzle region. All dimensions are normalized with respect to the 


\begin{tabular}{|r|r|}
\hline \multicolumn{2}{|r|}{$Y=M 0+M 1^{*} x+\ldots M 8^{8} x+M 9^{*} x^{9}$} \\
\hline$M 0$ & $1.34894 \mathrm{e}-1$ \\
\hline$M 1$ & $-7.57597 \mathrm{e}-5$ \\
\hline $\mathrm{M} 2$ & $-1.2157 \mathrm{e}-5$ \\
\hline $\mathrm{M} 3$ & $1.02414 \mathrm{e}-7$ \\
\hline $\mathrm{M} 4$ & $-2.65543 \mathrm{e}-10$ \\
\hline $\mathrm{M} 5$ & $2.17603 \mathrm{e}-13$ \\
\hline $\mathrm{R}^{2}$ & $9.87239 \mathrm{e}-1$ \\
\hline
\end{tabular}

Figure 7. Polynomial fit coefficients for the maximum displacement as a function of the membrane frequency

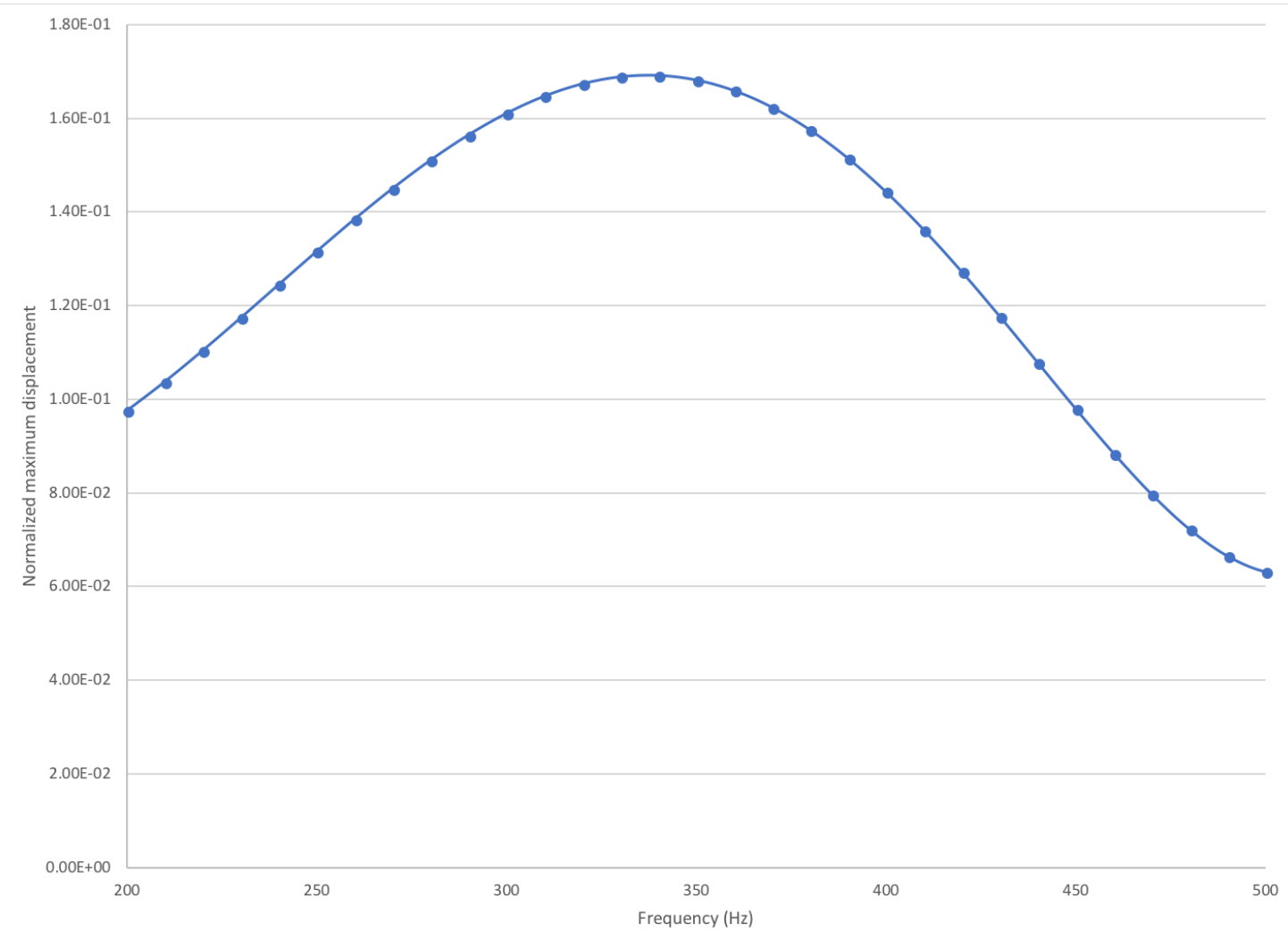

Figure 8. Plot of the polynomial fit for the maximum displacement as a function of the membrane frequency

nozzle's width. The cavity is composed of two membranes of radius $R_{m}=20$ separated by a distance $h_{c}=1$, and are both contained in the x-z plane. The nozzle connects the cavity to the outside box and is 
characterized by its height $h_{n}=4$ and an opening of length $l_{n}=12$ and width $d_{n}=1$ that is perpendicular to the y-axis. The outside region models the quiescent flow outside the actuator and is made of an elliptical cylinder (cylinder with elliptic cross section). The major and minor radii of the elliptical cross section are 42 and 30, respectively, and the height of the elliptic cylinder is set to 34. The shape of the outside box was designed to account for the rotation of the synthetic jet downstream of a rectangular nozzle reported in the published literature. The synthetic jet becomes wider in a plane that is parallel to the membrane motion (left view of Fig. 9). The computer-aided design (CAD) model of the actuator created with Cubit is shown in Fig. 9. The left view corresponds to a plane parallel to the membrane motion. The center view is a 3D view of the geometry. The right view shows a plane perpendicular to the membrane motion. The three regions - the outside box, the nozzle, and the cavity - are labeled for clarity. The membrane of interest is also labeled in the left view of Fig. 9. Note that the cylinders inside the outside box were used to help in the meshing process and thus are not part of the geometry per se.

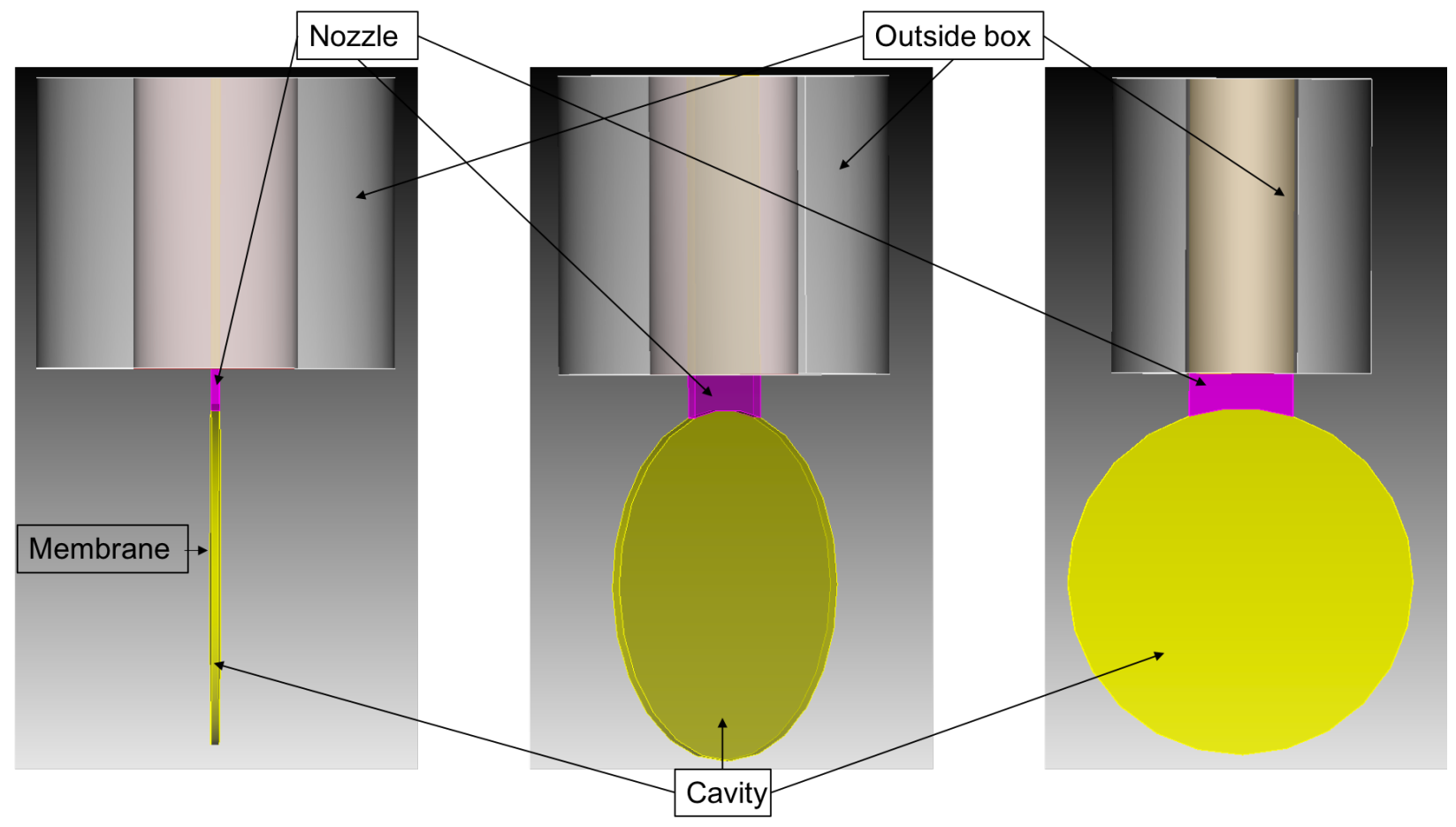

Figure 9. CAD model of an actuator.

The computational grid for this project was also generated with Cubit and is shown in Fig. 10. Since the Reynolds number is defined with respect to the nozzle's width, the nozzle was first meshed by supplying an adequate mesh size (Fig. 11). The mesh in the cavity was built by extruding the mesh of the membrane mesh generated with the Submap scheme. Note that the mesh density decreases as it moves towards the bottom of the cavity, which is explained by early investigations showing that most of the flow motion occurs in the top half of the cavity. The outside box was meshed with the Hole scheme available in Cubit and was extruded in the y direction with a bias scheme. After setting element types to HEX27, the mesh was exported in a Exodus format and imported in Nek5000 with the exo2nek tool. 


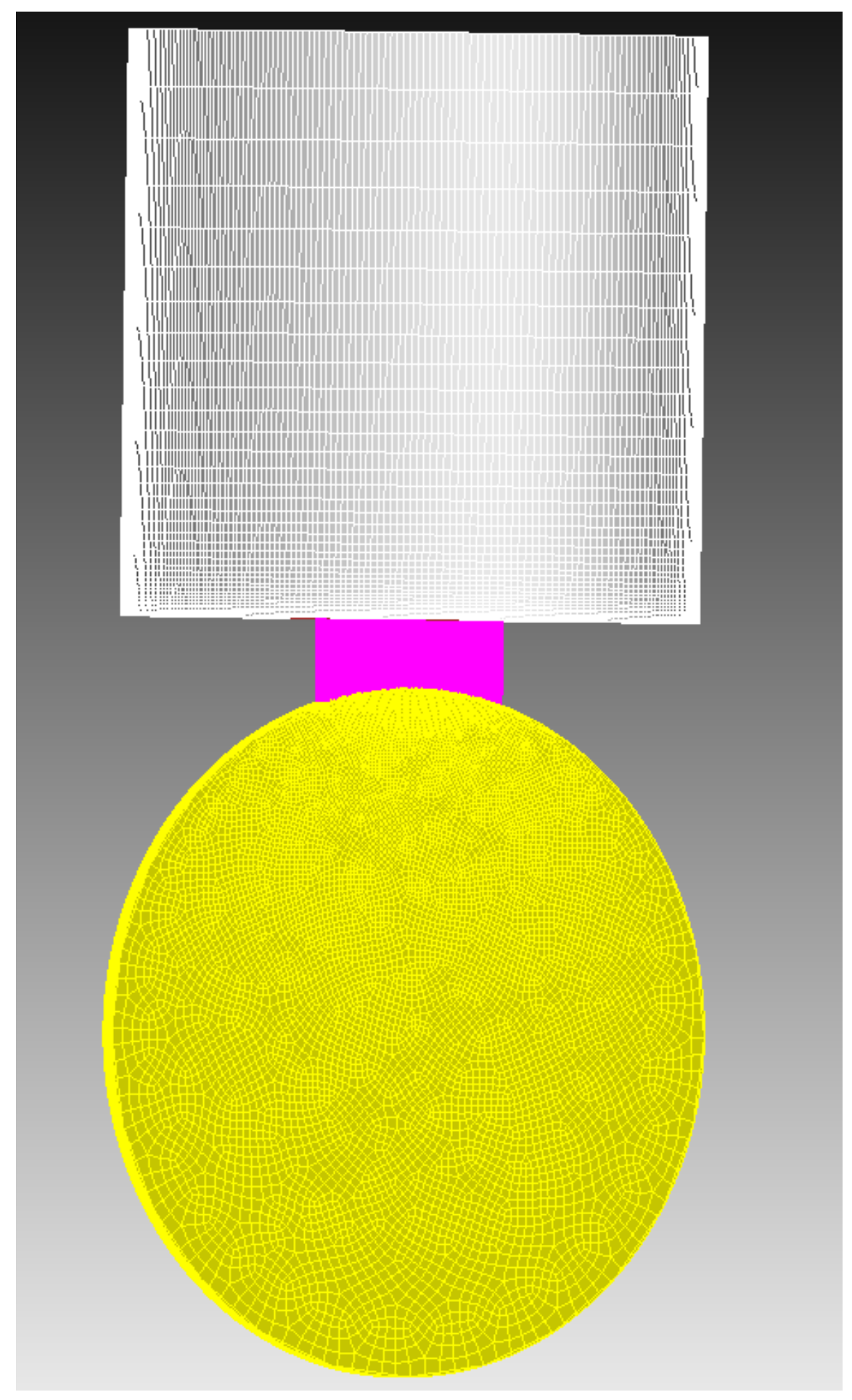

Figure 10. Full computational grid of an actuator. 


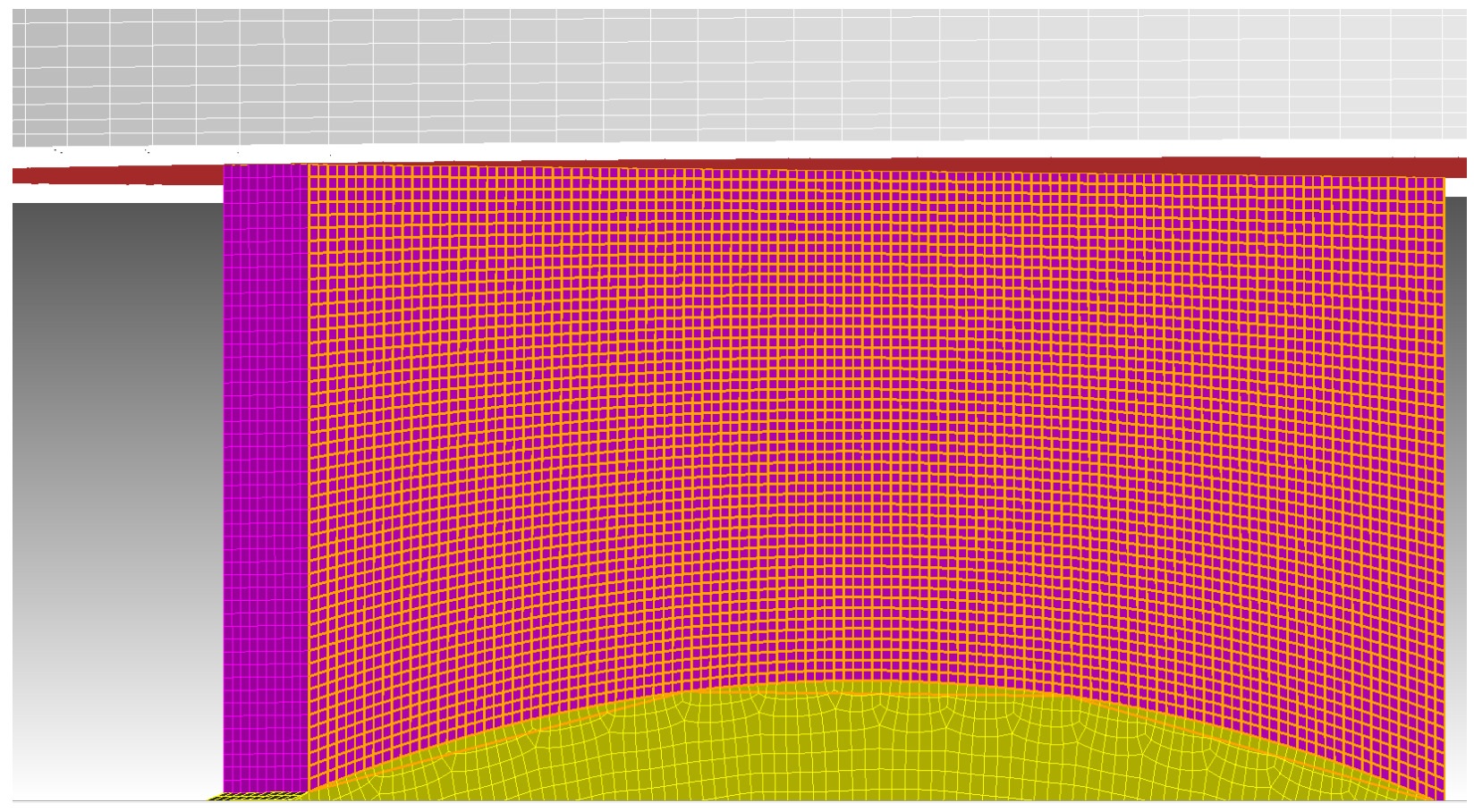

Figure 11. Zoom-in of the computational mesh in the nozzle region.

\subsubsection{Initial conditions}

The initial conditions consisted of a fluid at rest and a flat membrane in the cavity. Restart files were also used to collect time-averaged data which requires to start from a fully developed flow.

\subsection{DEFINITION AND AVERAGING}

DNSs of turbulent flows can be used to benchmark numerical solutions obtained with low-order models. A common example among the published literature is to benchmark a URANS or RANS numerical solutions with DNS data by tuning some of the turbulent model coefficients. In principle, benchmarking is a simple concept, but it requires great care in practice. DNS solution cannot be used firsthand; it must be post-processed at run-time or after the run finishes. The most common approach is to time average the DNS instantaneous field to produce a smooth field that can then be used to benchmark a low-order numerical solution. Time-averaging is a mathematical and statistical process that isolates the average flow behavior from the instantaneous DNS data and requires a sufficient number of time steps with respect to the flow parameters. Other averaging processes can be considered, such as volume-, surface- and line-averaging.

Because of the high computing cost of DNS and the large amount of data to store, it is vital to consider the variables that will be used for benchmarking. Note that such a list of variables is problem-dependent even though quantities such as the time-averaged velocity, the time-averaged Reynolds stresses, and the surface-averaged shear stress are commonly used.

Since the objective of this work is to benchmark a URANS solution of a synthetic jet produced by an actuator, the remainder of this section will exclusively focus on this benchmark. The geometry is divided 
into three regions: a cavity in which the two membranes move, an outside box, and a nozzle that connects the cavity to the outside box. To ensure the correctness of the benchmark process, the following questions must be answered:

1. what are the variables of interest?

2. what averaging method should be used?

3. which parts of the geometry are of interest?

Answers to these questions for the current study case are as follows:

- the time-averaged velocity to compare against experimental data.

- the time-averaged vorticity to compare against experimental data.

- the volume-averaged velocity in the nozzle and cavity.

- the surface-averaged velocity at the exit of the nozzle.

The flow in the actuator can be divided into cycles based on the periodic motion of the membrane. A cycle is defined herein as the time interval between two maximum displacements. Half a cycle is defined as the time interval between two extremum displacements. Time averaging will then be performed over an $\mathrm{N}$ number of cycles denoted by $T_{N}$ (6 to 10 cycles) to ensure good statistical validity. Quantities averaged over a volume $\mathrm{V}$ or a surface $\mathrm{S}$ are denoted by the symbol $<\cdot>_{V, S}$ in the following.

The statements provided above are defined mathematically :

- time-averaged velocity:

$$
\overline{u_{i}(\vec{x})}=\frac{1}{T_{N}} \int_{0}^{T_{N}} u_{i}^{\prime}(\vec{x}, t) d t \text { for } i=x, y, z
$$

- volume-averaged velocity in a volume V:

$$
<u_{i}(t)>_{V}=\frac{1}{V} \int_{V} u_{i}^{\prime}(\vec{x}, t) d V \text { for } i=x, y, z
$$

- $\quad$ surface-averaged velocity over a surface $\mathrm{S}$ and normal $\vec{n}$ :

$$
<\vec{u}(t)>_{S}=\frac{1}{S} \int_{S} \vec{u}^{\prime}(\vec{x}, t) \cdot \vec{n} d S \text { for } i=x, y, z
$$

- surface- and time-averaged shear stresses on a wall boundary in the nozzle: for the sake of this study, it is assumed that the stream-wise direction and the y-direction are aligned in the nozzle. A transverse cross section of the nozzle is then in the $\mathrm{x}-\mathrm{O}-\mathrm{z}$ plan. Because the flow changes direction in the nozzle over a cycle, time-averaging of the velocity would results in a value close to zero. Instead, time average is performed over a half cycle to ensure that the y-component of the velocity remains of same sign. Thus, time-averaging of a variable $f$ in the nozzle will be performed over a half cycle $\mathrm{T} / 2$ and denoted by $\bar{f}_{T / 2}$. Assuming that the shear stress $\tau_{w, \alpha}$ is computed on a wall boundary of index $\alpha$ and a surface of $S_{\alpha}$, it reads:

$$
\overline{<\tau_{w, \alpha}>_{S}}=\frac{1}{S_{\alpha}} \int_{S_{\alpha}}\left[\int_{0}^{T / 2} \tau_{w, \alpha} d t\right] d S
$$


Equivalently, the total the shear stress $\tau_{w}$ over all wall boundaries in the nozzle is defined as:

$$
\overline{<\tau_{w}>_{S}}=\frac{1}{S} \int_{S}\left[\int_{0}^{T / 2} \sum_{\alpha} \tau_{w, \alpha} d t\right] d S
$$

where $S=\sum_{\alpha} S_{\alpha}$ is the total surface over all wall boundaries.

The total shear stress can be used to compute a friction velocity $u_{\tau}=\sqrt{\frac{\tau_{w}}{\rho}}$, and then define a dimensionless velocity $u^{+}$and a dimensionless wall distance $y^{+}$to plot a law of the wall. Note that the friction velocity is commonly used to nondimensionalize the Reynolds stresses and other velocity-dependent quantities.

\subsection{USE OF DIRECT NUMERICAL SIMULATION DATA FOR BENCHMARKING}

This section presents high-resolution numerical results of the flow produced by an actuator using Nek5000 CFD code. High power computing (HPC) capabilities were required to run the CFD model within a reasonable amount of time and with a resolution high enough to model small features of the turbulent flow. The mesh was generated with Cubit (see Section 3.7.1) and converted in a binary Nek5000 format using exo2nek Nek5000 tool. Boundary and initial conditions were implemented in the *.usr file, along with the logic to time-averaged data over multiple cycles. Data of interest are the drag in the nozzle, the time-averaged velocity in the nozzle, and at the exit of the nozzle. As the flow is unsteady, the first step involved obtaining a fully developed flow by running four cycles. The resulting numerical solution was saved in a restart file and later used as initial conditions for runs that collect data. Data presented in this section are all non-dimensional and were collected after the initial four cycles and time-averaged over six cycles which was found to be sufficient in some experiments conducted at the early stage of this project.

Numerical results of the drag in the nozzle, time-averaged data, and volume-averaged data are presented for the Reynolds and Strouhal numbers given in Table 5.

\begin{tabular}{|c|c|}
\hline Reynolds $\operatorname{Re}_{0}\left(U_{\max }\right)$ & Strouhal $S t_{0}\left(U_{\max }\right)$ \\
\hline 578 & $5.41 \mathrm{E}-002$ \\
\hline
\end{tabular}

Table 5. Case run with Nek5000.

\subsubsection{Time-averaged velocity field}

This section presents numerical results of the time-averaged velocity field for the case highlighted in Table 5. We first discuss the time-averaged velocity magnitude that is showed in Fig. 12 and 13. The time-averaged data were obtained by averaging over 6 cycles. The results show that the main shape of the synthetic jet is recovered. In the XY plane, the inverted triangular shape is recovered and the velocity magnitude reaches a maximum along a line that passes through the center of the actuator (jet centerline). In the YZ plane, a triangular shape at the exit of the nozzle $(y>24)$ is displayed. The time-averaged velocity profiles in the $\mathrm{XY}$ and $\mathrm{YZ}$ planes are consistent with the rotation of the synthetic jet downstream the nozzle exit observed in experiments. A closer look at the time-averaged velocity magnitude in the nozzle (Fig. 13) 
calls for a few remarks. The velocity reaches a maximum value at the edges that link the cavity to the nozzle. This reserve of momentum could be transferred to the synthetic jet by optimizing the nozzle shape for instance. Also, only the air in the top part $(y>10)$ of the cavity seem to actively participates in the overall flow.

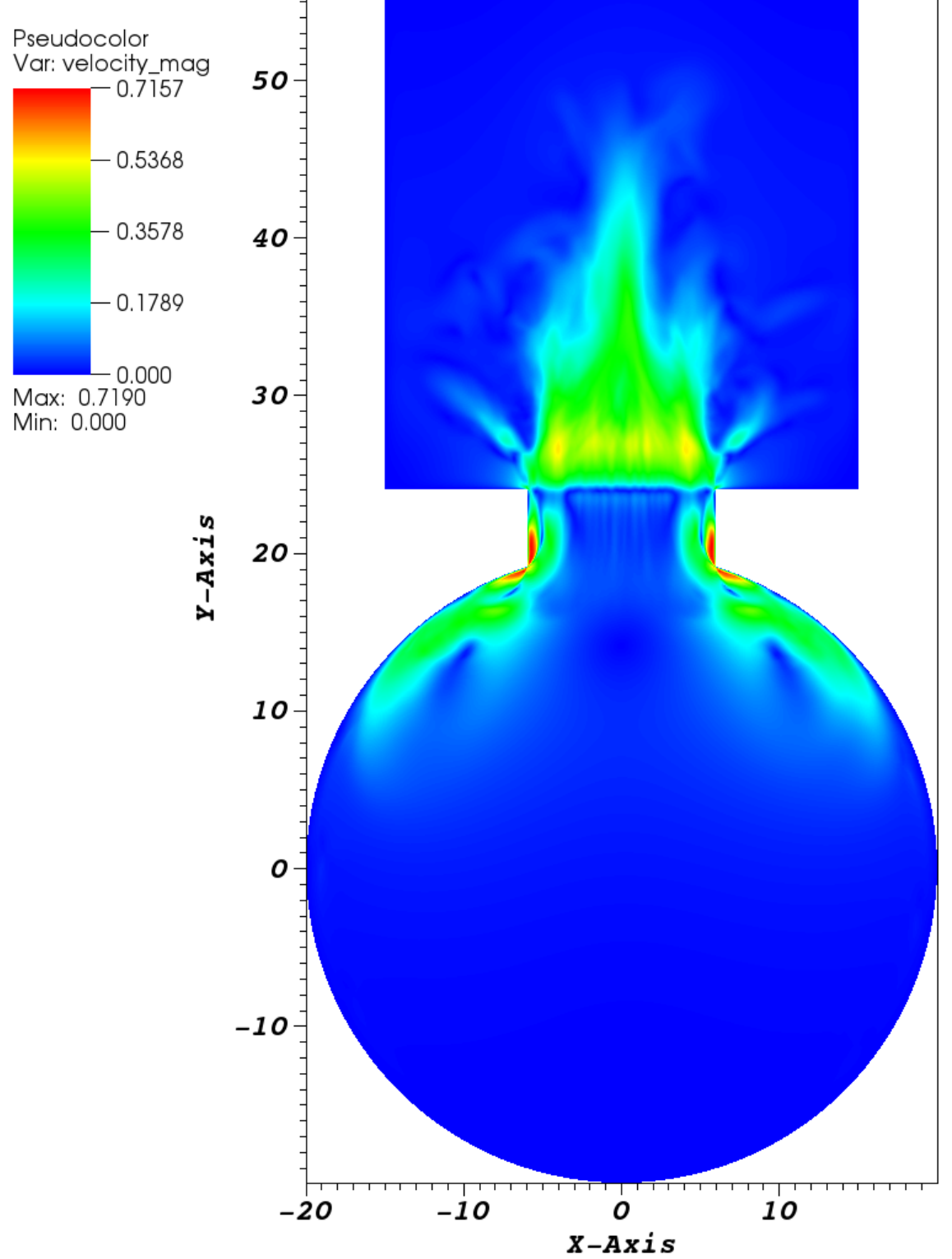

Figure 12. Time-averaged velocity magnitude over 6 cycles for case 1 in the XY plane. 


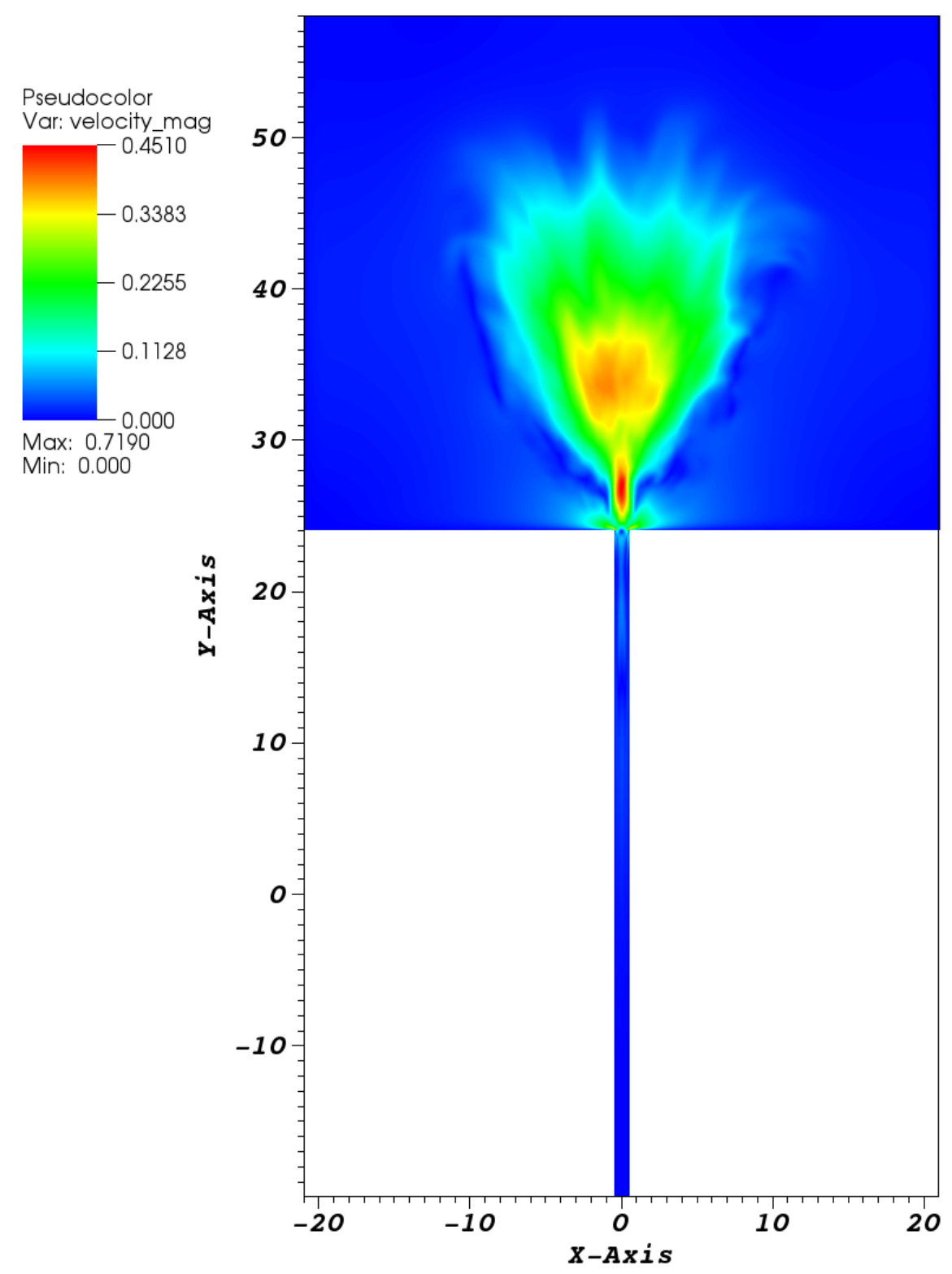

Figure 13. Time-averaged velocity magnitude over 6 cycles for case 1 in the YZ plane.

\subsubsection{Volume-averaged velocity in the nozzle and in the cavity}

We now analyze the plots of the volume-averaged velocity inside the nozzle and the cavity that is shown in Fig. 15a and Fig. 15b, respectively. The volume-averaged data of the velocity magnitude were computed on the fly using the definition provided in Eq. (30) and saved 36 times per cycle. The volume-averaged velocity plot displays periodic variations both in the cavity and the nozzle. The velocity in the nozzle experiences larger variation than in the cavity which is consistent with conservation of momentum for an incompressible model (the velocity changes between the nozzle and the cavity is function of the ratio of the nozzle volume to the cavity volume). In the nozzle, the volume-averaged velocity ranges from 0.2 to 0.95 

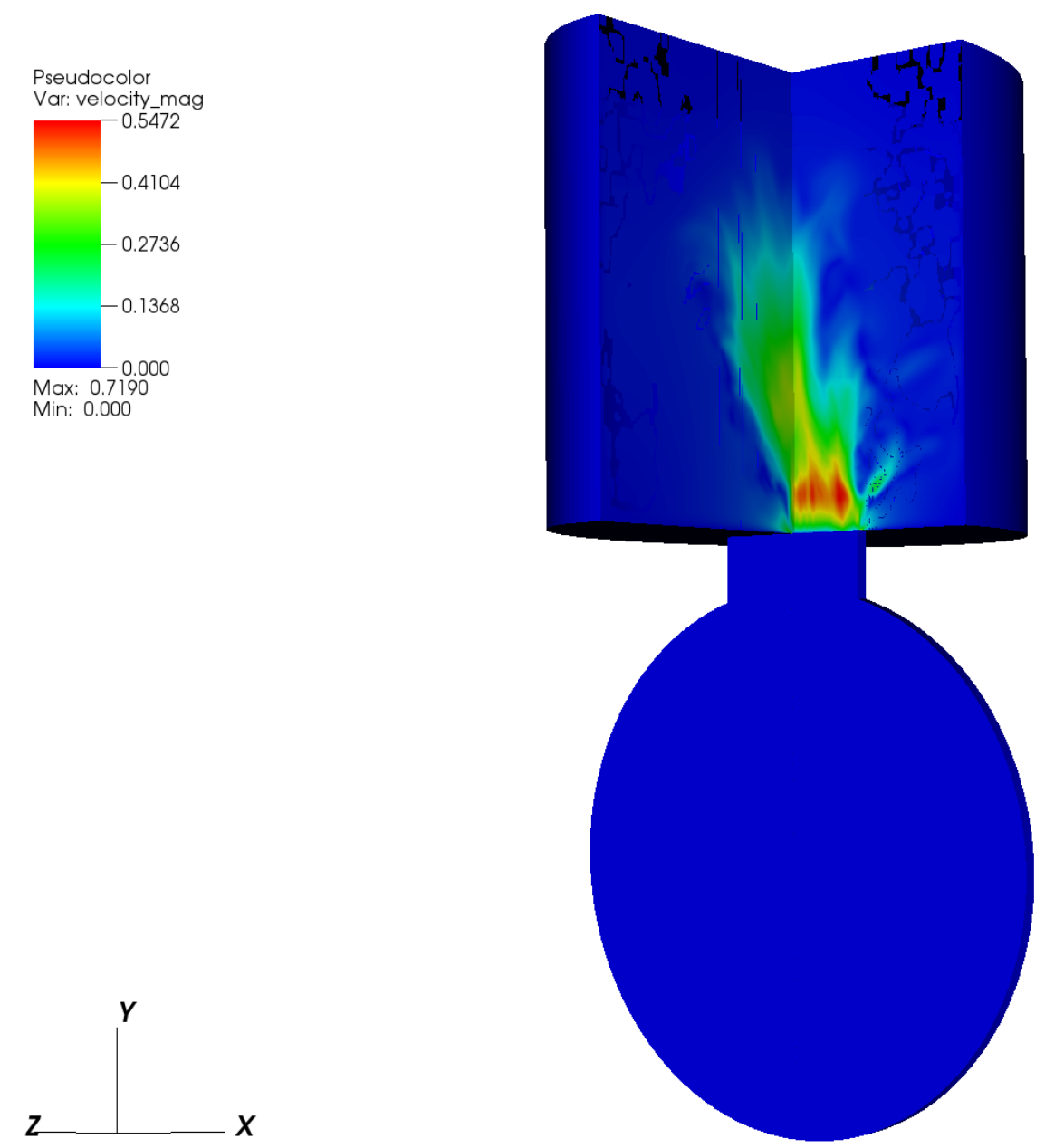

Figure 14. Time-averaged velocity magnitude over 6 cycles for case 1 in the YZ plane.

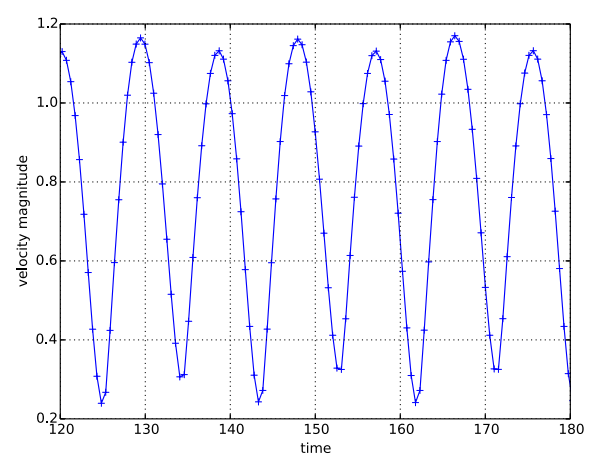

(a) Volume-averaged velocity field in the nozzle as a function of time.

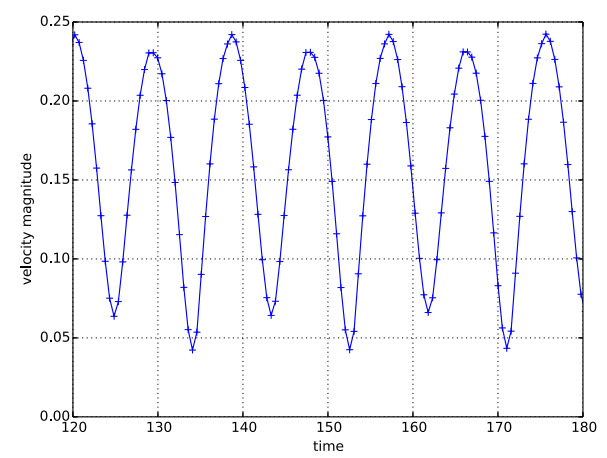

(b) Volume-averaged velocity field in the cavity as a function of time.

during a cycle. The velocity in the nozzle reaches a maximum value in the blowing or suction phase when the membrane reaches a extremum velocity. The minimum value is reached when the flow reverses, i.e, the 
membrane velocity changes sign.

\subsubsection{Drag in the nozzle}

The surface-averaged drag is first shown in Fig. 16 for all four walls in the nozzle. The wall indices are labeled from 1 to 4 . Indices 1 and 2 correspond to the walls in the $x-y$ plane and indices 3 and 4 correspond to walls in the $y-z$ plane. The wall drag was computed using the Nek5000 built-in function torque and saved 36 times per cycle in a text file. In the first half of the cycle $(t<185)$, the air flows out of the nozzle

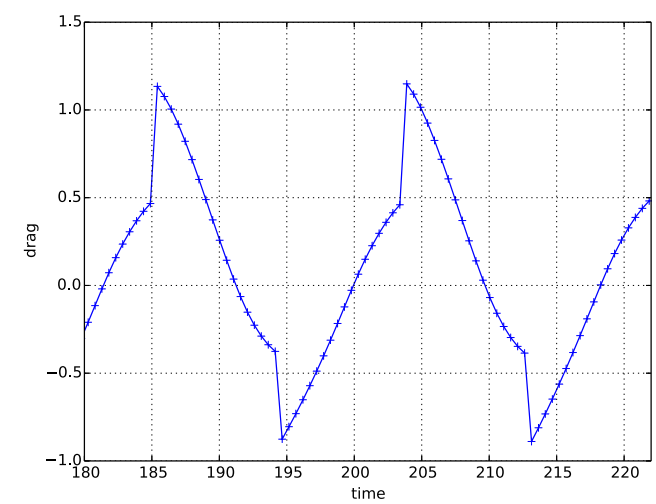

(a) Surface-averaged drag on wall 1.

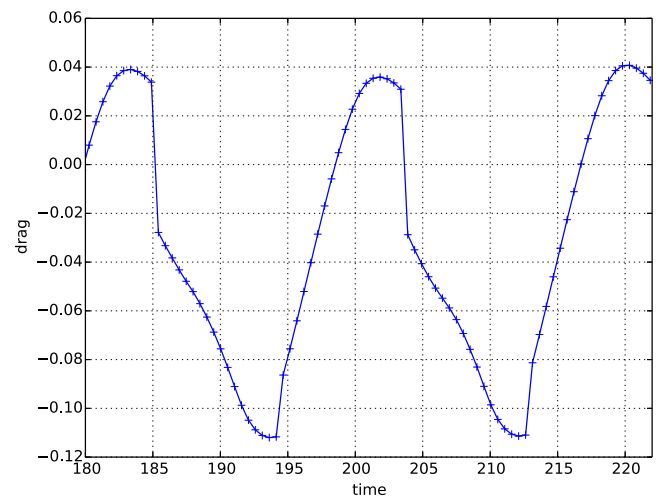

(c) Surface-averaged drag on wall 3.

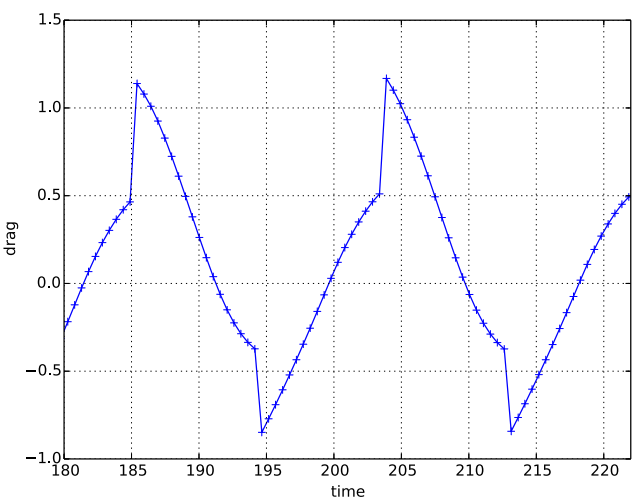

(b) Surface-averaged drag on wall 2.

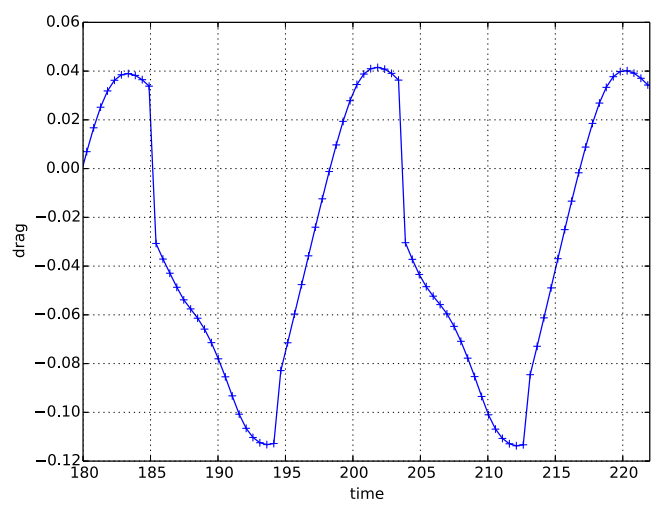

(d) Surface-averaged drag on wall 4.

Figure 16. Surface-averaged drag in the nozzle.

as the cavity volume decreases due to the membrane motion, which is defined as a positive wall drag. The wall drag first increases and then decreases during the first half of the cycle. This is consistent with the membrane velocity that decreases with time to reach zero as $t=T / 2$. The initial high-velocity of the membrane causes the air flow and the drag to increase. As the velocity membrane decreases, the air velocity and drag also decrease. When the second half-cycle starts, the air flow reverses as the cavity volume increases causing the drag to change sign. Over a full cycle, the drag variations are periodic. The same variations are observed for the wall drags computed on all four walls. It is also noted that the wall 
drag for indices 1 and 2 , and indices 3 and 4 yield the same values, respectively, which suggests that the flow is symmetric with respect to the $\mathrm{x}-\mathrm{z}$ and $\mathrm{x}-\mathrm{y}$ planes.

\begin{tabular}{|c|c|c|}
\hline Cycle & drag in first-half of cycle (blowing) & drag in second-half of cycle (suction) \\
\hline 1 & -0.374 & 0.469 \\
\hline 2 & -0.377 & 0.462 \\
\hline 3 & -0.387 & 0.459 \\
\hline
\end{tabular}

Table 6. Time and surface-averaged drag on nozzle wall for case 1.

\section{SUBJECT INVENTIONS}

No inventions have been developed under this CRADA

\section{COMMERCIALIZATION POSSIBILITIES}

The proposed technology aims to solve the following problems that will lead to the practical deployment of synthetic jet actuators:

- Heavy-duty trucks account for just $4 \%$ of all registered vehicles on the road in the United States but use $25 \%$ of all road-fuel and emit $25 \%$ of all greenhouse gases caused by transportation. Air resistance accounts for more than 50\% of fuel the consumed at highway speeds and can reach $20 \%$ of all operating expenses of a typical US fleet. The aerodynamic drag is heavily impacted by side winds. For example, a side wind of as low as $5 \mathrm{mph}$ can increase the vehicle's fuel consumption by up to $10 \%$.

- The safety and efficiency of trucks is highly dependent on constantly fluctuating environmental, operational, and climatic conditions.

- Despite these operating variables, truck aerodynamics is mostly addressed using permanent structural surfaces (truck exteriors, add-on deflectors) that are articulated to address a small portion of combinations of the above-mentioned conditions.

- State-of-the-art knowledge in vehicle aerodynamics is limited, and despite the significant effects of air resistance and changing wind conditions on stability and efficiency, the ability of most aerodynamic devices to adapt to these conditions is very limited.

- Therefore, the large gap between a truck's aerodynamic design requirements and the real-world conditions within which it operates is a problem impacting safety and efficiency of trucks.

\section{PLANS FOR FUTURE COLLABORATION}

Based on the accumulated expertise in performing HPC simulations of synthetic jets, the industry has expressed interest to work in the future with ORNL in the following areas: 


\subsection{A COMPUTATIONAL FRAMEWORK FOR MODELING THE APPLICATION OF ACTIVE SLOW CONTROL TO REDUCING TRANSMISSION LOSS OF VISION SENSORS ON SEMI AND FULLY AUTONOMOUS VEHICLES}

CleanJet is a fluidic windshield wiper which uses AFC technology to maintain visual clarity of vision sensors embedded in semi-autonomous and fully autonomous vehicles (AV) for all environmental and weather conditions. To increase driving safety, the CleanJet uses electric actuators that generate strong, data-driven synthetic air jets using very little power to maintain optical surfaces such as camera and Lidar lenses clean. The air jets can adapt their responses (air sweeping, vectoring, various air jet unsteady structures, etc.) autonomously based on driving conditions. Ultimately, the success of this project will pave the way to increase the safety and efficiency through utilization increase of AV. Actasys has already performed preliminary proof of concept (PoC) tests demonstrating AFC capabilities to maintain a large surface as clear under simulated harsh environmental and weather conditions. Considering the interaction between the air jets with different types of particles, the project combines an experimental path led by Actasys and a computational path led by ORNL. Actasys is currently collaborating with leading automotive OEMs, Tier 1s and Lidar companies to advance development and for testing performance under real life conditions.

\subsection{ROBUST MODELING ENVIRONMENT FOR DESIGNING AND OPTIMIZING SYNTHETIC JET ACTUATORS}

To enable widespread application of AFC technology on commercial transports, Actasys Inc. (Actasys), in collaboration with The Center for Advanced of Multifunctional Material Systems at University of California, Los Angeles (CAMMS-UCLA), and ORNL intend to develop a model-based environment for the advancement of design and performance validation of AFC using synthetic jet actuators (AFCSJ). The core objective of this approach is establishing a feedback loop between new computational models, lab tests, and field experiments in order to mature AFC actuation system design in a time-efficient, cost-effective, and ready-implementable manner. This is a significant improvement on the current prevailing approach of iterative build-and-test for AFC development.

\subsection{A COMPUTATIONAL FRAMEWORK FOR DEPLOYING ADAPTIVE AUTONOMOUS AERODYNAMICS ON TRUCKS (CADAT)}

The proposed concept is a computational framework for deploying adaptive autonomous aerodynamics on trucks (CADAT). CADAT is intended for designing and analysis of performance of such systems when integrated into truck designs under various operational conditions. The computational framework, developed by ORNL, combines CFD modeling with high-reliability dynamic controls, coupled with machine learning. Adaptive aerodynamic systems to be simulated using CADAT use AFC techniques and devices developed by Actasys to adapt truck aerodynamics to operational scenarios such as platooning, weather conditions such as side winds, ambient temperature and humidity, and road and load conditions. The deployment strategy of adaptive aerodynamics and the design space definition is done in collaboration with the trucking industry. CADAT calibration and validation is performed through full scale track testing.

\section{CONCLUSIONS}

This project successfully modeled a synthetic jet produced by an actuator using computational dynamics fluid models using Star-CCM+ and Nek5000. The numerical results showed good agreement with 
experimental data provided by Actasys. Insights on how to optimize the actuator were recovered by performing a sensitivity analysis of the time-averaged velocity to the cavity height and the frequency using HEEDS software.

High-fidelity solutions obtained with Nek5000 showed the CFD model was able to predict the main features of the synthetic jet. Further investigations will be needed to obtained a better time-averaged solution: longer time-average or higher polynomial order to increase the mesh resolution. Both options will require significant HPC ressources that are currently not available.

This project was mutually beneficial to Actasys and ORNL national laboratory. The sensitivity analysis will help Actasys to optimize their actuator design. The ORNL team gained valuable experience in CFD modeling that will be of use for future HPC4Mfg projets.

\section{References}

[1] M Deville, P Fisher, and E. Mund. High-order Methods for Incompressible Fluid Flow. Cambridge University Press, New York, 2002.

[2] P Fisher, Lottes J.W., and G Kerkermeier S. Nek5000 web page, 2008.

[3] L.W. Ho. A Legendre spectral element method for simulation of incompressible unsteady viscous free-surface flows. PhD thesis, MIT, Boston, U.S.A, 1989.

[4] M. Schmitt, C.E. Frouzakis, A.G. Tomboulides, Y.M. Wright, and K. Boulouchos. Direct numerical simulation of multiple cycles in a valve/piston assembly. Phys. Fluid, 26, 2014. 035105. 
Final Report Certification

for

CRADA Number NFE-17-06595

\section{Between}

UT-Battelle, LLC

and

Actasys, Inc.

(Participant)

\section{Instructions:}

Mark the appropriate statement in 1a or $1 \mathrm{~b}$ below with an 'IX." Refer to the articles in the CRADA terms and conditions governing the identification and marking of Protected CRADA Information (PCI).

If no $\mathrm{PCl}$ is identified, the report will be distributed without restriction. If $\mathrm{PCl}$ is identified, the report distribution will be limited in accordance with the CRADA terms and conditions governing release of data. In all cases items 2 and 3 must be true. That is, the report cannot contain Proprietary Information and a disclosure must be filed prior to release of the report.

This certification may either be made by using this form or may be made on company letterhead if the Participant desires. A faxed copy of this completed form is acceptable.

The following certification is made for the subject final report:

1. (a) $\square$ The final report contains information that qualifies as "Protected CRADA Information" (PCI). The PCl legend is printed on the report cover, and the $\mathrm{PCl}$ is clearly identified.

OR

(b) $\boldsymbol{x}$ The final report does not contain "Protected CRADA Information." The "Approved for Public Release" legend is printed on the report cover.

2. The final report does not contain Proprietary Information.

3. By the signature below, the Participant has no objection to the public distribution of the final report due to patentable information.

For the Participant:

David Menicovich

(Name)

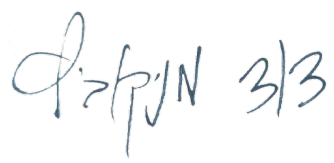

Cofounder, VP Product

(Title)

$10 / 25 / 2018$

(Date) 